\title{
Integrating RTI case management within LGU Health Centers: An intervention study
}

Marilou P. Costello

Carmeli Marie Chaves

Chona R. Echavez

Jesus Encena

Jocelyn Ilagan

See next page for additional authors

Follow this and additional works at: https://knowledgecommons.popcouncil.org/departments_sbsr-rh

Part of the Health Services Research Commons

How does access to this work benefit you? Let us know!

\section{Recommended Citation}

Costello, Marilou P., Carmeli Marie Chaves, Chona R. Echavez, Jesus Encena, Jocelyn Ilagan, and Orlanda Pagulayan. 1998. "Integrating RTI case management within LGU Health Centers: An intervention study," final report. Manila: Population Council. 
Authors

Marilou P. Costello, Carmeli Marie Chaves, Chona R. Echavez, Jesus Encena, Jocelyn Ilagan, and Orlando Pagulayan 


\title{
PHILIPPINES
}

\section{INTEGRATING RTI CASE MANAGEMENT WITHIN LGU HEALTH CENTERS: AN INTERVENTION STUDY}

\author{
Marilou P. Costello, Ph.D. \\ Carmeli Marie Chaves, M.A. \\ Chona R. Echavez, Ph.D. \\ Jesus Encena, M.D. \\ Jocelyn Ilagan, M.D. \\ Orlando Pagulayan, M.D.
}

\section{Final Report}

ASIA \& NEAR EAST OPERATIONS RESEARCH AND

TECHNICAL ASSISTANCE PROJECT

FAMILY PLANNING OPERATIONS RESEARCH AND

TRAINING (FPORT) PROGRAM

Population Council, Manila

in collaboration with

the Department of Health

USAID Contract No. DPE-C-00-90-0002-10

Strategies for Improving Family Planning Service Delivery

July 1998

\section{ACKNOWLEDGMENTS}


This project was conducted by Population Council-Manila, in collaboration with the Department of Health under the ANE OR/TA Project with support from the Office of Population, US Agency for International Development, Contract No. DPE-C-00-90-0002-10. The research team acknowledges the support and cooperation of the following in the successful conduct of the RTI Integration Study:

Department of Health Secretary, Hon. Carmencita Reodica; Assistant Secretary and IFPMHP Manager Dr. Rebecca B. Infantado; Assistant Secretary and UHNP Coordinator Dr. Nemesio Gako; Dr. Loreto Roquero, and Dr. Jojo Aricheta of the Family Planning Service; Dr. Marlene Borromeo, Dr. Teody Wi, Dr. Renee Faldas of the STD/AIDS Unit; and Dr. Rebecca Ramos of the Women's Health and Nutrition Project;

Mayor Ismael Mathay ( Quezon City), Mayor Pablo Magtajas (Cagayan de Oro City), Mayor Paulino Emano (Tagoloan Municipality), and Mayor Huberto Paurom (Jasaan Municipality) for allowing the research to be conducted in their administrative areas and for continuing to provide logistical and financial support to RTI integration in their health clinics during and even beyond the study period;

Project collaborators in the regional, provincial, city, and municipal health offices, such as Dr. Marietta Fuentes, Dr. Avelino Grospe, Dr. Edith Abocejo, and Dr. Jose Obenza of DOH Region X; Dr. Patricia Lim and Dr. Gracebel Angeles of the Misamis Oriental Provincial Health Office; Dr. Magdalena Ybanez and Dr. Perla Dosayla of the Quezon City Health Office; Dr. Rico Jacutin, Dr. Erickson San Juan, Dr. Rachel Dilla, Dr. Mila Eugenio, and Dr. Belen Ligo of Cagayan de Oro City Health Office; and Dr. Jose Acaylar and Dr. Edilberto Morelos of the Northern Mindanao Medical Center (NMMC);

The consultants for the RTI training: Dr. Ilya Abellanosa of the Cebu City Health Office; Dr. Maripaz Nazareno of DOH-UNFPA; Dr. Ruth Leoncio of DOH; Sol Atayan and Rhea Ravanera of DKT International; Aida Santos of WEDPRO; and Judy Ipanag of NMMC;

The offices who gave logistical support: Reachout AIDS through Jomar Fleras for 
sharing their STI brochures and videotapes; Medichem Pharmaceuticals through Willy Verzosa and Johnny Penalosa for donating RTI drugs; Program for Appropriate Technology in Health through Drs. Carmina Aquino and Joan Castro for sharing their IEC materials; Philippine Daily Inquirer and Sunstar Daily through Froilan Gallardo and Lina Sagaral for their press coverage and write-ups; the University of the Philippines Population Institute through Dr. Aurora Perez, and Florio Arguillas for his assistance in data processing; the UP Psychology Department, particularly Professors Cecilia Conaco and Mariano Sto. Domingo for conducting the focus group discussions;

Collaborators from the Research Institute for Mindanao Culture: Dr. Michael Costello, Prof. Lita Sealza, Dr. Magdalena Cabaraban, Lourdes Wong, Edwina Lagos for their technical and logistical back-up for the monitoring activities in Cagayan de Oro and Misamis Oriental;

Finally, we are especially indebted to all the service providers in the seven clinics who participated in this research and the men and women who consented to be part of the study. 


\section{TABLE OF CONTENTS}

Acknowledgments

List of Acronyms Used

V

List of Figures

vii

List of Tables

Executive Summary

Introduction

Background

Objectives

ix

$\mathrm{X}$

Methodology

Program Intervention

Research Design

Data Collection

Focus Group Discussions

Observations of Health Center Activities

Situation Analysis and Client Records

Cost Analysis

Results and Discussion

Focus Group Discussions

Improvement in RTI Diagnostic Facilities and Services

Improvement in Service Providers RTI Knowledge,

Diagnostic Skills and Information Provision

Client Satisfaction

RTI Case Management

Cost Analysis

Conclusions and Lessons Learned

References

Appendices
A: RTI MIS Forms
B: Drugs Used in Syndromic Management
C: Participating Health Centers
D: RTI Brochure 


\section{E. Costing Data Assumptions and Worksheets}

\section{LIST OF ACRONYMS USED}

\begin{tabular}{|c|c|}
\hline AIDS & Acquired Immuno-Deficiency Syndrome \\
\hline AIDSCAP & AIDS Control and Prevention Project \\
\hline ANE OR/TA & Asia and Near East Operations Research and Technical Assistance \\
\hline BHS & Barangay Health Station \\
\hline DMPA & Depo Medroxy Progesterone Acetate \\
\hline $\mathrm{DOH}$ & Department of Health \\
\hline FGD & Focus Group Discussion \\
\hline FP & Family Planning \\
\hline FPORT & Family Planning Operations Research and Training \\
\hline \multirow[t]{2}{*}{ HIV/AIDS } & Human Immuno-Deficiency Virus/Acquired \\
\hline & Immuno-Deficiency Syndrome \\
\hline ICPD & International Conference on Population and Development \\
\hline IEC & Information, Education, Communication \\
\hline IUD & Intra-uterine Device \\
\hline \multirow[t]{2}{*}{ JHPIEGO } & Johns Hopkins Program for International Education \\
\hline & in Reproductive Health \\
\hline $\mathrm{KOH}$ & Potassium hydroxide \\
\hline LGU & Local Government Unit \\
\hline LPP & Local Performance Program \\
\hline $\mathrm{MCH}$ & Maternal and Child Health \\
\hline MI & Macro International \\
\hline MIS & Management Information System \\
\hline NGO & Non-Government Organization \\
\hline NSO & National Statistics Office \\
\hline NSS & Normal Saline Solution \\
\hline OR & Operations Research \\
\hline PATH & Program for Appropriate Technology \\
\hline $\mathrm{PE}$ & Physical Examination \\
\hline PID & Pelvic Inflammatory Disease \\
\hline POGS & Philippine Obstetric and Gynecologic Society \\
\hline PPO & Population Program Officer \\
\hline RA & Risk Assessment \\
\hline
\end{tabular}


RTI

SA

SDP

SM

STD

UHNP

UTI

VDRL

WHO

WHO-GPA
Reproductive Tract Infection

Situation Analysis

Service Delivery Point

Syndromic Management

Sexually Transmitted Disease

Urban Health and Nutrition Project

Urinary Tract Infection

Venereal Disease Research Laboratory

World Health Organization

World Health Organization - Global Program for AIDS 


\section{LIST OF FIGURES}

Fig.1 Conceptual Framework of the Relationships between the Intervention Program and Reduction of RTIs

Fig.2 Clients' Perception of Length of Waiting Time

Fig.3 Clients' Perception of Length of time for Consultation

Fig.4 Clients' Perception Whether There Was Enough Privacy

At the Clinic during Consultation

Fig.5

Proportion of Clients' According to Whether They Were

Advised by the Service Provider on RTI Prevention and Treatment

Fig.6 RTI Cases Identified in the Study Sites, May-October 1997

Fig.7 Percent of RTI Clients by sex

Fig.8 Percent of RTI Clients by Marital Status

Fig.9 Percent of RTI Clients by Occupation

Fig.10 Percent of RTI Clients by Number of Children

Fig.11 Method of RTI Case Finding, Female Clients, May-October 1997

Fig.12 RTI Clients by Incidental Findings

Fig.13 RTI Clients by Methods of Case Finding

Fig.14 RTI Clients by Chief Complaint

Fig.15 RTI Clients by Presenting Symptoms 
Fig.16 Current FP Method Used by RTI Clients

Fig.17 Current FP Users Among RTI Clients

40

Fig.18 Percentage of Laboratory Exams Conducted by Type of Exam

Fig.19 Drugs Provision for RTI Clients 


\section{LIST OF TABLES}

Page

Table 1 Reserach Design of the RTI Integration Study

Table 2 Annual Cost of RTI Case Management at a Clinic Without a Laboratory

Table 2.1 Cost for Various Annual Estimates of Patients in Clinic Without a Laboratory

Table 3 Cost of RTI Case Management at a Clinic with a

Laboratory

Table 3.1 Cost of Various Annual Estimates of Patients in Clinics with a Laboratory

Table 4Comparison Between Model 1 and Model 2 for 100

Patients 


\section{EXECUTIVE SUMMARY}

As a response to the ICPD Plan of Action calling for a more client-centered reproductive health approach, the Department of Health (DOH) through its Family Planning Operations Research and Training (FPORT) program, with technical and financial support from the Population Council (ANE OR/TA) studied the feasibility of integrating reproductive tract infection (RTI) services within selected public sector primary health care settings. Feasibility is defined as the ability of the intervention program to improve RTI diagnostic facilities and services, and to improve service providers' knowledge, diagnostic skills and the provision of information on RTIs.

RTIs include a variety of bacterial, viral, and protozoal infections of the lower and upper reproductive tract of both sexes. There are three common causes of these infections: (a) iatrogenic infections, which are related to inadequate medical procedures, such as unsafe abortions; (b) endogenous infections, due to the overgrowth of organisms normally found in the genital tract and may be associated with inadequate personal, sexual and menstrual hygiene, and (c) sexually transmitted infections (STIs).

In 1994, the DOH convened a committee to study the possibility of adopting the syndromic management of RTIs in the Philippines. Syndromic management is based on identifying a syndrome-- a group of symptoms and easily recognised signs--associated with a number of well-defined etiologies. Once a syndrome has been identified, treatment can be provided for the majority of the organisms responsible for that syndrome. Several syndromes can be managed easily and rapidly using clinical flowcharts (also known as algorithms) for diagnoses and treatment. Clinical flowcharts rationalize and standardize clinical decisionmaking. Their use can also standardize diagnosis, treatment and referral. In resource-poor settings, this approach is recommended since it allows health care providers to make a diagnoses without sophisticated laboratory tests.

The main objective of this operations research study was to describe and assess the implications of integrating RTI services within the Family Planning/Maternal and Child Health (FP/MCH) program. Following the newly developed STD management guide developed by DOH-AIDSCAP, and in anticipation of a nationwide program implementation, the Family 
Planning Service-DOH, through the FPORT undertook the lead in undertaking operations research to integrate RTI services in the FP/MCH programs of devolved primary health clinics. An intervention study was therefore developed to introduce syndromic management in seven study clinics. The seven clinics were: Bernardo and Toro Hills Health Centers in Quezon City; Lapasan, Carmen, and Macabalan Health Centers in Cagayan de Oro City; and Tagoloan and Jasaan Rural Health Units in Misamis Oriental Province. Four of these clinics (Jasaan, Macabalan, Lapasan and Carmen ) with existing laboratory facilities were upgraded in order for them to carry out simple microscopy and lab tests to supplement the syndromic approach. It had been recommended that flowcharts that include inexpensive and simple lab tests improve the ability to predict upper genital tract infections. The physicians, nurses and midwives in the clinics served as partners in this study by being in charge of filling out the study protocols which were developed to also serve as RTI records to be kept confidentially in their clinics.

A similar set of control clinics was identified in Bugo, (Cagayan de Oro City) Balingasag, (Misamis Oriental Province and Novaliches (Quezon City) for comparative analysis.

The research methodology employed was a qualitative analysis of local health-seeking behaviour and existing RTI case management through focus group discussions and interviews with key informants. Baseline data were obtained by the study team composed of a social scientist and a physician trained in STI management using the situation analyses (SA) tools before the intervention activities began. To determine the change in the readiness of the health centers to provide RTI services, a second SA was conducted three months after the last facilitative supervision was held. Four standard SA protocols were used: (1) an inventory of clinic facilities and clinic records, (2) staff interview, (3) observation of client-provider interaction, and (4) client exit interview. In addition, cost data were gathered and analyzed to determine how much it would cost to integrate RTI services.

The findings of the baseline data were used to develop and define the intervention plans for integrating RTI case management within the FP/MCH programs. The major intervention activities consisted of the following: (1) training of service providers (physicians, nurses and midwives) assigned in the clinics of the intervention areas on RTI case management, including a three-day refresher course for medical technologists; (2) upgrading of clinic 
facilities and laboratory capability and ensuring the availability of drugs in the intervention clinics providing RTI services; (3) post-training facilitative supervision and provision of technical assistance on developing and maintaining an integrated RTI/FP management information system; (4) development of RTI educational materials to be used by clinic personnel to increase community awareness about the prevention, detection, and treatment of RTIs, including information on where to obtain RTI services in the community.

The focus group discussions revealed that knowledge levels among men and women in the community about RTI symptoms and prevention were moderate, while popular treatment regimens were based on non-scientific traditional practices. (A separate report of the FGDs has been prepared entitled "Beliefs and Practices Regarding Reproductive Tract Infections in the Philippines"). In general, participants' knowledge about RTIs is limited to STDs. Infections brought about by iatrogenic or endogenous causes were rarely, if at all, mentioned. In addition, there seems to be a generally unfavorable attitude toward condom use, as their use is linked with the practice of patronizing commercial sex workers. Misconceptions about certain FP methods were also widespread.

Some complaints about public sector health centers (compared to private clinics) were voiced, including the opinion that the public health providers are not too competent and sometimes less accommodating, long waiting time in the health centers, and lack of privacy during consultations. The participants noted that it is not uncommon to entertain suspicions of marital infidelity when a spouse is diagnosed to have an STD. It was also mentioned that since problems on communicating with the spouse often arise at times like these, it is important that service providers know how to deal with the sensitive nature of RTIs. Likewise, the importance of maintaining privacy in the consultation was underscored.

The pre-and post-intervention SA results showed that the health centers' readiness to provide RTI services improved significantly after the intervention. Health providers previously offering only FP/MCH -related services were managing RTI cases along with the other services they provide. History taking and risk assessment for STDs were routinely done by health providers, including midwives. A private area for consultation was already provided by most clinics, and clinics with laboratory facilities conducted simple microscopy and lab tests in support of RTI diagnosis. Staff reported that they now manage RTI cases in the clinic with 
more confidence. More service providers in SA2 said that they included the topic of RTIs in their health talks, including the availability of RTI services in their health center. In addition, clients reported that they were told by the service providers about RTI signs and symptoms, and their prevention and treatment, which was not the case during the first SA exit interviews.

Within the six-month observation period, 243 RTI cases were managed in the seven health centers. The typical RTI client was found to be female, 31 years old, married, and was either a housewife or an office clerk/employee. The majority of their partners (husbands/wives) were construction/factory workers and drivers. The average RTI client had 2.8 children. More than half of the female clients were walk-in clients, while about twenty percent of women diagnosed with RTIs were asymptomatic and were diagnosed after a regular FP procedure or check-up. A substantial number (18\%) were referred from other SDPs, particularly from the barangay health stations with RTI trained service providers.

The analysis of risk assessment results showed that a total of $31 \%$ of RTI clients showed positive risk assessments. Thirteen percent of the clients believed that their partner may have had other (another) sex partner in the last two months; 9\% believed their partner had an STD or had been treated for an STD, 7\% admitted having had more than one sex partner while $2 \%$ changed sex partners in the last three months.

Fifty-one percent of the RTI clients were family planning users, using IUD (23\%), pill (15\%) and DMPA (6\%). Tests to determine RTI (KOH, NSS, Gram stain) were done in $31 \%$ of all RTI cases. Pap smear was taken in $18 \%$ of the cases. These tests were done by trained medical technologists in the health center and findings were used to support clinical assessments.

The chief complaint among women who came to the clinics was vaginal discharge (60\%), which is also the most common presenting symptom. The most common RTIs found were: vaginitis and/or cervicitis (60\%), trichomoniasis and /or candidiasis (17\%), and gonorrhea and/or chlamydia (10\%). A substantial proportion (75\%) of this was managed using syndromic approach without the aid of laboratory tests. The remainder of these cases were managed based on the client's medical history, physical exam, and laboratory test (12\%), and on history and physical exam only (8\%). RTI management appeared to be generally 
satisfactory, as most clients were reported as having received treatment and instructions for treatment compliance and given counseling and health education messages. Condom use was also encouraged. The majority were also asked to return for a follow-up visit. Clinic records showed that $74 \%$ of these clients returned for a revisit, of which $66 \%$ were later found to be symptom-free.

The cost analysis showed that the cost per RTI client in a clinic without a laboratory is about $\$ 14$ while the cost per client in a clinic with a laboratory is $\$ 16$. The provision of RTI services in clinics with laboratory facilities entails higher fixed costs. In clinics without laboratories, the bulk of the cost lies in treatment costs. These findings imply that RTI management supplemented with simple lab test and microscopy appears to be more costeffective as the number of clients increase.

This study has shown that it is indeed feasible to integrate RTI prevention and management in FP/MCH clinics. It has been said that health providers in the Philippines are overburdened by the many health programs that they are handling. This observation lead to the idea that adding a new service will meet resistance from health providers. However, this observation was not borne out by this study. Other questions raised about RTI integration include the following: (1) the fear of spreading STDs to FP/MCH clients because of the use of the same set of instruments for examining RTI clients; (2) resistance of physicians to allow their nurses and midwives to prescribe RTI medicines to patients; (3) the fear of driving away "regular" FP/MCH clients because they are afraid of being stigmatized and mistaken as commercial sex workers. All these issues surfaced during the training and were discussed extensively.

During RTI management training the participants reached a consensus on how to deal with these issues. Health providers were unanimous in expressing their satisfaction with the RTI integration initiatives in their clinics. Six months after the study, they were found to still be managing RTI cases. As of this writing all six clinics continue to receive some form of support for drugs from their local government.

Although the project succeeded in integrating RTI services within LGU health centers, this program will continue to present numerous challenges to policymakers and program 
managers. Partner management; sustaining RTI drug provision; improving the counseling skills of service providers, and the provision of a separate counseling room to ensure visual and auditory privacy in the clinics, will need to be adequately addressed in order for this service to be truly institutionalized in the health centers.

The main lessons learned from this intervention study are the need for post-training facilitative supervision and the need for a standing order from the medical officer for the service providers, particularly the nurses and midwives, to manage RTI cases with greater skill and confidence. The high rate of return visits of RTI clients is indicative of the success of RTI integration. The high rate of return visits was attributed to the consistent counseling of the health providers and the follow-up visits made by the community volunteer workers. Consistent program efforts to implement regular FP check-ups is an effective strategy for screening RTI-asymptomatic women. Finally, it was learned that involving LGU officials (i.e. mayors, municipal health officers, etc.) at every step of the intervention process can significantly help to sustain RTI integration efforts. 


\section{Integrating Reproductive Tract Infection Services Within the FP/MCH Program of LGU Health Centers}

\section{INTRODUCTION}

\section{Background}

This operations research project was conceived in response to the need of the Department of Health (DOH), Government of the Philippines to implement the ICPD Plan of Action. The call in Cairo for a client-centered reproductive health approach includes, along with other services, the management of reproductive tract infections (RTIs).

Numerous studies have shown that reproductive tract infections are extremely widespread among women from the less developed world (Younis, et al., 1993; Wasserheit, et al., 1989; Bang, et al., 1989). Similar problems have been shown to exist in the Philippines. Sanchez and Juarez (1994) reported, for example, that more than a quarter of Davao City women in the reproductive ages suffer from one or another forms of RTIs. It also appears that women frequently do not seek treatment for these conditions. One reason for this is that RTI clients are often asymptomatic. Even when this is not the case, a medical consultation may be foregone because of the culturally sensitive nature of this illness. The 1993 Philippine Safe Motherhood Survey reported that nearly two-thirds of women suffering from abnormal vaginal discharge did not seek care as they did not think it was serious enough to warrant a visit to the health center (National Statistics Office and Macro International, Inc., 1994).

Studies have also shown that the adoption of some methods of FP -- in particular, IUDs and tubal ligation -- is significantly associated with RTIs (e.g., Younis et al., 1993; Bhatia and Cleland, 1995). IUD users have been reported to be about two to five times more likely to develop PID than women not using a contraceptive method. Thus, RTIs can work to decrease the demand for contraception as well as to bring about FP discontinuation once symptoms are perceived to be side effects of contraceptive use. Many studies have shown that the most common reason women give for discontinuing a contraceptive method is the perception or fear of a side effect. In the Philippines, 40 percent of pill users and 22 percent of IUD users discontinued use within a year of adoption because of side effects and health concerns (NSO and MI, 1994). Regardless of whether there is a relationship, users often 
attribute any symptom, particularly any reproductive tract symptom, to their method of contraception. In the absence of accurate diagnosis and effective education and treatment, women will most likely blame their vaginal discharge on their contraceptive method rather than consider the possibility of her husband's infidelity (Ward, 1996).

On the other hand, potential FP clients who exhibit RTI symptoms may be temporarily refused service (particularly in the case of ligation or IUD) until their infection has been treated. A history of PID and the presence of cervical infection are contraindications to IUD use. It is thus clear that the provision of RTI services can be an important complement to existing family planning/MCH programs.

Based on epidemiological data about STDs in the Western Pacific Region, the World Health Organization developed the syndromic approach and later endorsed it for STD case management in resource-poor settings. Syndromic management makes use of flowcharts or algorithms so that health providers simply have to follow directions for treatment based on the patient's signs and symptoms as well as his or her risk assessment results. This approach is said to be optimal for at least four distinct reasons, namely, its simplicity, cost-effectiveness, ability to provide early diagnosis and treatment, and applicability to most primary health care settings.

In 1994 the Department of Health convened a committee to study the possibility of adopting the syndromic management of RTIs in the Philippines. A series of training workshops for social hygiene clinic physicians in the country was conducted beginning in February 1995 at the Research Institute for Tropical Medicine. The training centered on syndromic management, using modules developed by WHO. This approach is said to be optimal for at least four distinct reasons, namely, its simplicity, cost-effectiveness, ability to provide early diagnosis and treatment, and applicability to most primary health care settings.

A subsequent outgrowth of these activities was the development of a standard STD management guide developed by DOH-AIDSCAP, which was officially launched and endorsed by the DOH in 1996.

The syndromic management (SM) guidelines developed for the Philippines include algorithms for six of the most common syndromes or presenting symptoms encountered in 
clinical practice: urethral discharge, vaginal discharge, genital ulcer disease in men and women, scrotal swelling, lower abdominal pain, and eye infection in the newborn. There are three algorithms for syndromes in women (two for vaginal discharge and one for lower abdominal pain), two for syndromes in men (urethral discharge and scrotal swelling), one for a syndrome found in both men and women (genital ulcers), and one for newborns (eye infection).

The choice of drugs recommended in the National STD Management Guidelines was based on the latest results of local drug sensitivity studies. The syndromic approach makes use of standardized treatment, using the most effective medications available. It also recommends the use of single dose therapy in order to improve drug compliance and minimize the emergence of resistant organisms.

Although training of service providers in the syndromic management of RTIs in many parts of the country have already been held, the experience of adding RTI case management in LGU public health centers and integrating this within the constellation of health services offered has not been studied.

It is not certain that this type of management can be feasibly introduced given the concerns about overburdened health providers who continue to handle several programs of the Department of Health. In this context, how will health providers view this added task? Additionally, given this situation, questions have been raised as to whether or not this integration effort will just serve to weaken the existing FP/MCH programs.

Another consideration has to do with the social stigma attached to STDs, the more well-known RTIs. In the present system, STD cases are handled in social hygiene clinics which are often attached to city health clinics and have been widely known to mainly serve the needs of commercial sex workers (CSWs). Some may argue that to introduce RTI case management in a FP/MCH health center could lead to a loss of regular clients out of fear social stigma. The question, therefore, is whether this will happen in clinics that provide RTI services.

In the context of devolution and limited local resources, it remains problematic to convince local officials to prioritize health programs if these concerns compete with other programs (e.g. infrastructures--roads, school buildings, etc.) which are perceived to be more 
important and immediate.

\section{Study Objectives}

The major objective of this operations research study was to assess the feasibility of an intervention to integrate RTI prevention and services in the FP/MCH program. Feasibility is defined as the ability of the intervention program to improve RTI diagnostic facilities and services, and to improve service providers' knowledge, diagnostic skills and the provision of information on RTIs. These improvements are expected to contribute to greater client satisfaction with RTI services, and more effective case management of RTIs (see Figure I).

A second objective of the study was to assess the cost of integrating RTI prevention and services into the FP/MCH program. This is particularly important because primary health care is currently being provided by devolved local government units (LGUs). LGU officials will not support the programmatic change required if integration proves successful without being able to assess its financial implications. 


\section{METHODOLOGY}

\section{Program Intervention}

The intervention program consisted of four components. Each of these components is discussed below.

\section{Training of Service Providers in RTI Case Management}

The training of service providers in RTI case management was a major feature of the intervention. A total of seven physicians, six nurses, and 29 midwives were given a five-day training in RTI case management. The training enhanced the health providers' ability to detect signs and symptoms of RTIs, their mode of transmission and their treatment. The main reference material used in this training was the Training Manual on Care and Prevention of Sexually Transmitted Diseases and its accompanying STD Case Management Guidelines, both of which were endorsed by the Department of Health. These manuals were developed by AIDSCAP for use in the Philippine setting and were based on the WHO/GPA syndromic flowcharts. Consultants from the Family Planning Service and the STD/AIDS Units of the DOH, the DOH Region X Office, LGU Social Hygiene Clinics, and DKT International trained the clinic staff.

The training format included both didactic and practicum approaches. History taking, risk assessment, diagnosis, treatment, and counseling procedures were the major topics covered in the didactic portion while the practicum emphasized role playing to establish competency. Sessions on integrated RTI/FP/MCH counseling and community awareness were emphasized to guide the service providers to include appropriate RTI messages and counseling to the standard FP/MCH messages already being given to clients in the clinic and in the community (See report on the RTI Case Management Training, Lauremar Hotel, Cagayan de Oro City, March 1997).

The open forum sessions revealed preconceived notions and initial confusion held by the service providers regarding RTI management. The discussions centered on the hesitancy of 
service providers to manage commercial sex workers with STDs along with "regular housewives"; whether nurses and midwives should be allowed to provide treatment for RTIs; and the lack of RTI drugs. Concerns were also raised on how to tell the client about sensitive matters related to RTIs/STDs during counseling and how to convince her sex partner to come to the clinic for treatment.

Four medical technologists (from four study sites with laboratory capability) also underwent a three-day practicum refresher course at the Northern Mindanao Medical Center in Cagayan de Oro City on basic laboratory methods ( wet mounts and Gram stains) for the detection of RTIs.

\section{Upgrading of Clinic Facilities and Laboratory Capability}

The second component of the intervention involved the upgrading of facilities and laboratory equipment within the clinics. Based on the findings of the baseline data analysis, several clinics were found to lack the basic facilities (e.g., a private area for counseling), equipment (specula, sterilizers, etc.) and reagents needed for simple laboratory tests. On a need basis, equipment was provided to the clinic sites. Basic drugs recommended under the national STD management guidelines were also made available for the duration of the study by the Department of Health, local governments, and donations from drug companies solicited by the $\mathrm{DOH}$ and the study team members.

A refresher course in simple microscopy and laboratory testing was held to enhance competency in conducting laboratory tests required for RTI management. This training followed closely the curriculum on laboratory procedures developed by JHPIEGO for its Genital Tract Infection Training in 1995.

\section{Technical Assistance on MIS and Facilitative Supervision}

A third component of the intervention consisted of activities geared to assist service providers in maintaining the standards for proper care management within the 
clinic setting. Health personnel were provided technical assistance in designing management information forms for all RTI cases managed in the clinics. These RTI records were placed in a folder separate from the regular client records to maintain full confidentiality. These forms had an added function in that they served as research protocols for the study. Informed consent forms were also kept in the same folder.

The RTI individual treatment form was designed in such a way that it can be filled in in the order of a standard RTI consultation procedure, from recording client information to scheduling a follow-up visit. Thus, as the provider performs the steps of a regular consultation, the form is simultaneously completed.

A member of the study team who is a physician made regular visits to all participating clinics in order to provide post-training supportive supervision. This last activity had the major benefit of enabling clinic staff, particularly the nurses and midwives, to be more confident in diagnosing and treating RTI cases. This was viewed as crucial in areas that had a heavy client load or in clinics where the physician reported only weekly, therefore leaving the task of managing RTI clients mainly to nurses and midwives.

\section{Community Awareness and Education}

A fourth activity consisted of increasing community awareness of RTIs. Service providers were encouraged to include the topic of RTI in their regular health talks, such as in pre-clinic lectures, "bench talks", mothers'ffathers' classes, lectures to food handlers, as well as in regular FP counseling. They were given information guidelines covering the following topics: symptoms and consequences of RTIs, care procedures (e.g., treatment compliance, partner diagnosis and treatment), and the availability of RTI services at the health center.

Moreover, community health volunteers were oriented at their respective health centers by the physician in charge of the unit. These community volunteers were involved in raising community awareness about the availability of RTI services at the clinics.

The project developed three types of IEC materials for the community education program. These are: an RTI brochure written in the local dialect which was given out to clients during FP counseling and other health talks (see Appendix D); a syndromic management

poster adopted from the National STD Management Guidelines, and a guide on the 4Cs 
(Counseling, Compliance, Condom Use, Contact Tracing), both for the provider's easy reference. 
Figure 1. --Conceptual Framework of the Relationship Between the Intervention Program and Reduction of RTIs

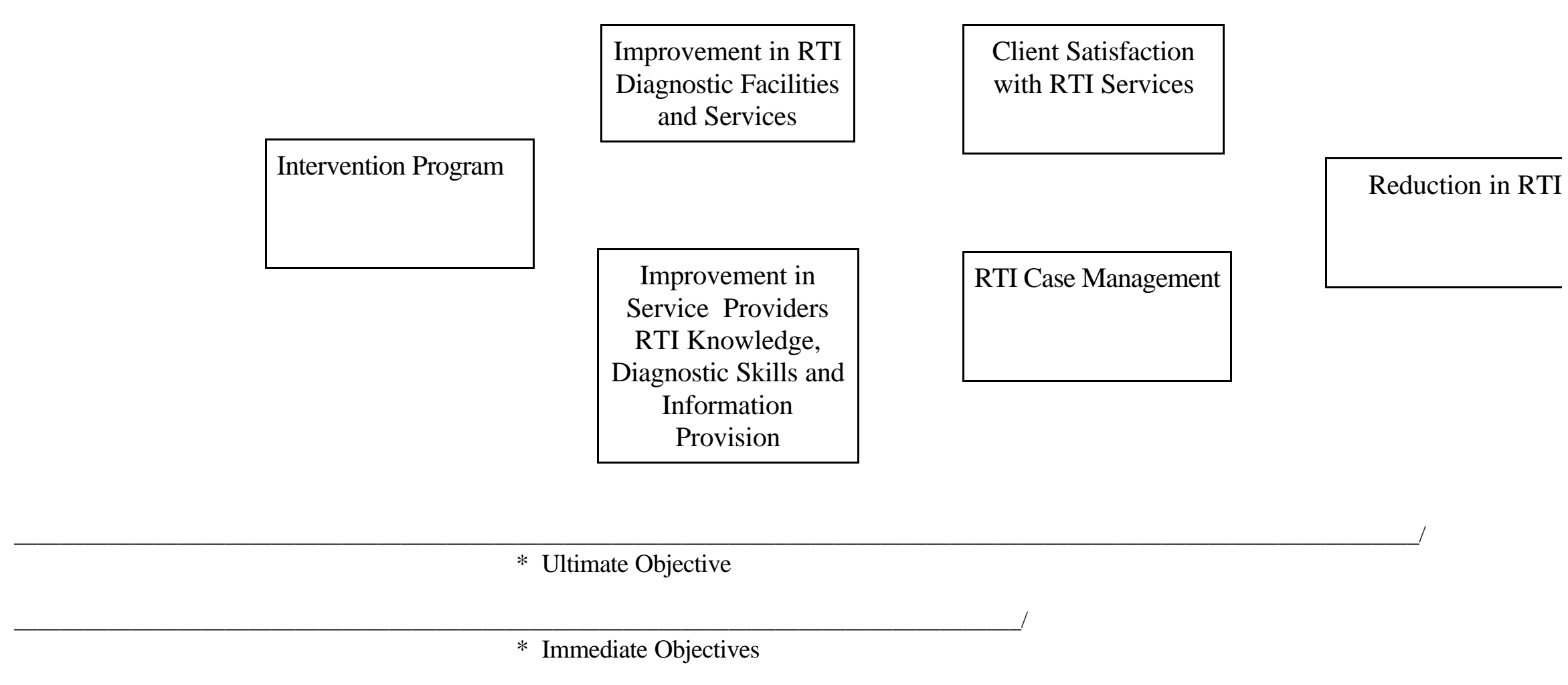




\section{Research Design}

This study utilizes a post-test only control group design with the added feature of baseline data collection for the experimental areas (health centers). The following table summarizes the design:

Table 1. Research Design of the RTI Integration Study

\begin{tabular}{|l|l|l|l|l|}
\hline Areas & $\begin{array}{l}\text { Focus Groups } \\
\text { and } \\
\text { Observations }\end{array}$ & $\begin{array}{l}\text { Baseline } \\
\text { Situation } \\
\text { Analysis }\end{array}$ & $\begin{array}{l}\text { Program } \\
\text { Intervention }\end{array}$ & $\begin{array}{l}\text { Follow-up } \\
\text { Situation } \\
\text { Analysis }\end{array}$ \\
\hline $\begin{array}{l}\text { Experimental } \\
\text { Health Center }\end{array}$ & $\mathrm{x}$ & $\mathrm{x}$ & $\mathrm{x}$ & $\mathrm{x}$ \\
\hline $\begin{array}{l}\text { Control Health } \\
\text { Center }\end{array}$ & & & & $\mathrm{x}$ \\
\hline
\end{tabular}

Four sets of interrelated activities characterize the research design: focus groups and clinic observations; a baseline situation analysis; a program intervention lasting six months; and a follow-up situation analysis. Each of these activities is discussed in more detail under the sections on data collection and program intervention.

Seven health centers in three areas were chosen for the intervention study. These are: Quezon City (Bernardo and Toro Hills Health Centers), Cagayan de Oro City (Macabalan, Lapasan, and Carmen Health Centers); and Misamis Oriental Province (Tagoloan and Jasaan Health Centers). In addition, the catchment barangay health stations (BHS) under each of these centers were included.

In addition to their status as Local Performance Program (LPP) areas, these health centers were strategically chosen mainly for their OR capability (location near a research center with OR capability), their proximity to institutions with capability for STD management, as well as for their access to technical assistance from other agencies working on the same concerns, such as JHPIEGO, POGS, PATH, the STD/AIDS Unit of the DOH, and the World Bank-funded Urban Health and Nutrition Project. 
The three study areas exhibit different demographic characteristics. Quezon City is a large, densely populated urban area situated within the Metropolitan Manila Area. It operates two well-equipped social hygiene clinics (STD centers) serving between 5,000 to 12,000 clients every year, generally commercial sex workers. Cagayan de Oro is a typical mediumsized city located in Northeastern Mindanao. It is also one of three sites in the country chosen for participation in the national AIDS Surveillance Project as well as the Women's Health Project. Misamis Oriental, located also in Mindanao, represents a relatively low density area, basically rural province and is a pilot area for the Reproductive Health Program of the DOH. All three areas have been included in past OR capacity-building activities of the Family Planning Operations Research and Training (FPORT) Program being coordinated by the ANE OR/TA Project of the Population Council.

A similar set of control health centers were selected to provide comparisons with the experimental centers. These are the Bugo Health Center in Cagayan de Oro City, Balingasag Health Center in Misamis Oriental Province, and Novaliches Health Center in Quezon City.

\section{Data Collection}

Focus Group Discussions: Focus group discussions (FGDs) were held among men and women in the three study communities to understand local perceptions of RTIs and the treatment-seeking behavior of men and women. The FGDs were held separately for men and women in Quezon City, Cagayan de Oro, and Misamis Oriental. The discussions explored perceptions and attitudes about RTIs, social meanings of infections, commonly-sought treatment practices and other health-seeking behavior, knowledge of causes and consequences of RTIs, barriers to men's use of services, strategies for notifying partners and partner management, attitudes towards condom use, and perceptions about local patterns of gender relations.

Observations of Health Center Activities: Observations of health center activities were conducted, especially those which pertain to FP/MCH (e.g., prenatal care, IUD services) in 
general and RTI management in particular. Attention was given to the procedure that clients follow as they go through the different levels of health care providers (i.e. from midwife to physician) during their visit. In addition, in-depth interviews were conducted among all levels of service providers. The interviews covered the following topics: a) current clinic procedures for managing RTIs; b) experiences and perceptions of providers about the characteristics of RTI symptomatic clients as well as the management and treatment of sex partners; c) perceived local prevalence of RTIs; d) providers' self-assessment of their training needs.

Situation Analysis and Client Records: A modified situation analysis was conducted in all sites before the intervention was set up to determine the readiness of health clinics to provide RTI services. The study team assessed the availability and quality of facilities, equipment and personnel. Organizational and staffing patterns were also studied in order to understand better the roles and functions of clinic staff in the context of all other services and programs being provided in the clinic. The basic situation analysis instruments (Inventory of Clinic Supplies and Equipment, Staff Interview, Observation of Client-Provider Interaction, and Client Exit Interview) were used. A post-intervention situation analysis was conducted to assess any changes in the capability of the clinics to provide RTI services.

Data from client records for the year preceding the intervention were analyzed as part of the baseline information. An analysis was also made of the individual treatment records of RTI clients during and after the intervention. These RTI client forms were especially designed for the study and fulfilled the dual purpose of recording RTI client information and serving as research protocols for the study (see Annex A for a sample of the individual treatment record).

A situation analysis was also undertaken in three health centers (Novaliches, Bugo and Balingasag) where no intervention activities have taken place to serve as control areas for the study.

Cost Analysis: Cost data were gathered and analyzed to determine how much it would cost to integrate RTI services into the existing FP/MCH program of a primary health center. Costing of the line items was done for two types of clinics: A clinic with laboratory facilities and a clinic without such facilities. Questions relating to sustainability and replicability were 
also addressed.

\section{RESULTS AND DISCUSSION}

This portion of the report is presented in six sections: (1) focus group discussions; (2) improvement in diagnostic facilities and services; (3) improvement in service providers' knowledge, diagnostic skills and information provision; (4) client satisfaction; (5) RTI case management; and (6) cost analysis.

\section{Focus Group Discussions}

A more detailed description of the findings from the FGDs are contained in a separate report entitled "Beliefs and Practices About Reproductive Tract Infections: Findings from a Series of Philippine FGDs." The FGDs revealed that knowledge levels among men and women in the community about reproductive tract infections were moderate to moderately high at best. Preventive techniques were little understood while treatment regimens were in many cases based solely upon nonscientific, traditional practices. For example, treatment ranged from placing a "beef poultice" on the sex organ, "washing infected area with rubbing alcohol or vinegar," to "inserting the penis into a heated young coconut." In general, females knew more than males about the different types of RTIs. Discussions tended to focus first on those conditions of greatest personal concern to the group in question -- e.g., uterine infections and cancers among the women, STDs and AIDS for the men.

Uterine infections were stressed by the women, although they did not know the technical (scientific) name for these conditions. The symptoms they cited included fever, chills, and bleeding. Several alleged causes were mentioned, including an overly early return to sexual activity after the birth of a child, large family size (too many births weaken the uterus and make it prone to infection), use of the IUD, and having an abortion or wearing tight fitting pants.

Another RTI variety was described generally as itching of the female genitalia. Some 
women said they experienced this just before their menstruation, others just after. In the latter case the immediate cause was said to be either the menstrual blood or the use of sanitary napkins, which are hot and do not allow the genitalia to "breathe." Other causes cited were the use of unclean water for washing oneself, dirty underwear, allergy to birth control pills and use of the IUD.

All groups held extended discussions on STDs and AIDS. Among the older women, syphilis, gonorrhea and herpes were all mentioned but there seemed to be some confusion among the participants as to the exact distinctions among these diseases. Several men had not only heard about gonorrhea but had experienced it as well. They said that the major symptoms of this condition included pain in the abdomen and the penis, painful urination, and pus in the urine. A few women pointed out as well that STDs could result in infertility on their part or certain conditions affecting the newborn child.

The bulk of the discussants seemed to know only the sexually transmitted diseases as RTIs. The infections brought about by iatrogenic or endogenous causes were rarely, if at all, mentioned. There were some mention, however, of improper or unhygienic health habits that can cause RTIs.

Attitudes toward condom use were generally unfavorable except perhaps for a certain willingness to adopt these in cases where a commercial sex worker was being patronized. Rumors and misconceptions about the health consequences of adopting such modern FP methods as the IUD, oral contraceptives, and DMPA were often mentioned.

In addition, several specific complaints were lodged against the public health system. Most respondents had doubts about the competence of the health care workers associated with the LGU clinics. This was particularly true for BHS-level personnel (i.e., midwives) insofar as there was a strong preference for treatment by licensed doctors. Even in cases when a public sector doctor could be consulted, however, some doubts still remained.

Thus, comparisons between the quality of services of private health clinics and the public sector health centers were generally decided in favor of the former. Specific complaints mentioned with regard to the LGU clinics were that their personnel were less competent and less accommodating, that waiting times were longer, and that they could not offer sufficient 
privacy. Private practitioners were perceived as offering more in the way of privacy, confidentiality, and competency. The sole drawback in this case was, of course, that they also charge higher fees.

There was a tendency to assume marital infidelity when a married person was found to have contracted an STD. Because of this, many think that open interspousal communication is affected, with the men generally keeping the illness from their spouses. Most men admitted that they would prefer to deal with this issue by consulting a close friend or a doctor.

The risk assessment questions were presented to the FGD participants for their discussion and reaction. Reactions to the prospect of being subjected to the risk assessment questions were not negative. In fact many felt that they would not object to being asked these questions, because they perceive this to be part of the treatment. They were quick to add that this should be done in a confidential manner. For example, they insist that the interview must be conducted in private and the questions should be asked in a way that the sensitivity of the clients is considered, i.e., to minimize the chances that they would feel embarrassed or humiliated.

\section{Improvement in RTI Diagnostic Facilities and Services}

The FP/MCH and other services offered in the clinics were found to be quite satisfactory. Both SA1 and SA2 revealed that family planning services were available on a daily basis in all seven health centers. In some centers, however, clients were advised not to seek consultation on Wednesday mornings, because this is the day set aside for immunization. Depending on the volume of clients at the health center, clients were either seen upon arrival or had to wait for up to thirty minutes on the average before being seen by a service provider.

All clinics had piped running water, electricity, sufficient seating for clients, and working toilets and latrines. The examination areas were generally clean, had adequate water and lighting as well as auditory and visual privacy. A marked improvement was noted in the provision of a private area for counseling. Whereas in SA1 only two of the seven clinics had a private counseling area, after the intervention, SA2 results showed six of the seven clinics 
having such a facility. In the control group, two of the three health centers had a private room or area for counseling.

The intervention clinics showed a marked difference in the availability of a separate client record for RTI cases. While all the seven intervention clinics had a separate client record, no such record system was found in the three control clinics.

Four clinics in the study had a laboratory area, but the capacity of these laboratories was found in SA1 to be limited due to inadequate supplies, such as reagents and slides. None of the clinics could conduct basic laboratory tests for the detection of RTIs, although microscopes were available. Pap smears were collected in four of the seven health centers, although the readings were being done in government hospitals. Pregnancy tests were also performed but this depended on the availability of reagents.

The laboratory capacity of these clinics improved greatly in SA2, particularly in the capacity to conduct RTI tests. All four clinics had glass slides, cover slips, cotton applicators, droppers, alcohol lamps, a gram stain set, and other materials needed for basic lab tests for RTI (gram stains and wet mount).

Before the intervention, signs indicating the availability of FP services were rarely seen both inside and outside the building. After the intervention, all health centers had signs indicating the availability of FP services; in five clinics, signs could be found both outside and inside the health center. These aspects were not discussed in the RTI training but since this was included in the presentation of the first SA results, the providers took the initiative to ensure that signs were made visible outside and inside their health centers indicating availability of services. In addition, while the clinics had ample supplies of IEC materials such as flipcharts, samples of contraceptive promotional materials, posters, and brochures, only in SA2 were RTI brochures and posters available in all study clinics. No RTI brochure or poster was found in the control clinics.

In spite of the availability of materials, no health talks dealing with RTI was given in any of the clinics during the visit of the research team. In SA2, one health talk was given in one of the clinics in the intervention area during the visit of the study team. This does not mean, 
however, that health talks are rarely done. In interviews with the staff, at least three of the physicians reported that they regularly hold health talks in the community integrating RTI messages.

Family planning methods such as pills, condoms, DMPA injectables, and IUDs were generally available in the health centers. Records were kept in an orderly fashion, although some FP records were not completely filled out. In SA1, not all clinics kept separate records for RTI cases. This completely changed in SA2, where all seven clinics had separate records for RTI cases. In contrast, none among the control group had separate client records for RTI cases.

In SA2, all the study clinics managed RTI cases. These services were unavailable in these same clinics as shown in SA1 as well as in the control clinics in SA2.

\section{Improvement in Service Providers RTI Knowledge, Diagnostic Skills and Information Provision}

In SA1, although the majority of the service providers in the study had heard about RTIs, only three of the 22 providers interviewed had been previously trained in RTI case management. Three providers who were trained in RTI management felt however, that their training was inadequate in managing special cases. Almost all of the providers (21) said that they told clients who sought RTI services about other SDPs, particularly hospitals or STD clinics. In addition, materials for RTI diagnosis and treatment were unavailable.

The staff claimed that they encountered an average of one to five RTI cases per month, suggesting either a problem of underutilization of existing clinic services or an absence of RTI services in the clinics. The latter interpretation seems most plausible because of the fact that the health providers were generally not trained to manage RTI cases. Likewise, they did not perceive this to be part of the services generally offered in the primary health care setting.

The staff interview found that health providers tended to manage cases based only on their own particular clinical experience. No standard procedures were followed. Case 
management practices reported by staff varied from referrals (to STD clinics or to nearby hospitals and/or private physicians) to dispensing vaginal suppositories (when available) and/or prescribing antibiotic drugs. They also mentioned using albothyl "paint" to patients presenting with RTI related symptoms.

Pelvic examination was not routinely done. Others reported that they take client's history to give them an idea about the risks for contracting an STD. Moreover, midwives and nurses were found to be particularly concerned about the issue of prescription and dispensing of drugs, particularly in cases when the clinic physician was absent. Referrals to STD clinics are perceived by both clients and service providers as problematic because these facilities are associated with commercial sex workers and its stigmatizing nature prevents regular FP/MCH clients to seek services there.

This picture changed considerably in SA2, where all service providers reported having been trained in syndromic management. The majority of these providers (17) believed that the training they received was adequate for them to manage RTI cases in the clinic. They reported that they manage RTI cases routinely. On the other hand, no health provider in the control clinic claimed they could manage RTI cases. They generally tell women with symptoms to go to the hospital or to other facilities that they know provide these services.

The providers also showed a substantial increase in knowledge about RTI symptoms and the modes of transmission. The health staff agreed that sex with an infected partner was a mode of transmission. Some cited lack of personal hygiene, and a majority mentioned improper surgical or medical procedures as different ways in which STIs can be transmitted.

All twenty-two providers agreed that condoms offered protection against STDs. In SA1, sixteen providers mistakenly believed that pills offered protection against STDs. This number decreased to six in SA2.

Among RTI symptoms, vaginal discharge and lower abdominal pain were frequently cited by providers. A third condition, genital itchiness, was mentioned by close to half of the providers in SA2, but was not mentioned by any of the providers in SA 1.

More than half of the health providers (in SA2) believe that certain conditions had to be 
considered in allowing non-doctors, particularly nurses and midwives, to provide treatment after having been trained in RTI case management. One such factor was location of the clinic. According to many of these respondents, this approach might be acceptable in barangays that are rarely visited by physicians. Those who disagreed with this idea stressed that non-doctors cannot dispense drugs unless there is a "standing order" from the municipal health officer.

The interaction between providers and clients centered on the purpose of the client's visit, which was usually to obtain a FP method. In both SA1 and SA2, the majority of the clients came for follow-up visits, usually to have their next DMPA injection, to get a resupply of pills, or to ask about the possibility of switching to another FP method.

Observations of the client-provider interaction indicated that concerns about RTIs/STDs were not at all raised by either clients or service providers in SA1. The family planning service record normally used by the service providers included only a few items relating to RTI. But even this information was not consistently asked the client. In general, providers asked clients about their last menstrual period and their medical history but did not ask about either RTI symptoms or the client's sexual history. None of the providers discussed condom as a barrier method against STDs. Only three of the forty consultations mentioned condoms as a barrier method against RTIs/STDs.

In SA1, there were omissions on the part of providers during pelvic examinations. For instance, although all providers used clean/disposable gloves and a sterilized speculum, in only about half of the examinations did they wash their hands before the exam. Privacy was not always observed since in some cases persons other than the provider were present during a pelvic exam. In SA2, however, 14 of the 15 pelvic exams were conducted in a private area.

Although all clients were informed of the findings in SA1 less than half of them were informed before the pelvic exam about what to expect during the procedure. However, this number improved to three fifths of the clients in SA2.

Counseling and health education were received by the majority of clients in SA1 and SA2 on various topics related to FP/MCH. In SA1, topics included iron tablet supplementation, self-breast examination, basic information, the different FP methods, personal hygiene, breastfeeding, and use of condom as an alternative contraceptive. In SA2, aside from these topics, consultations also included RTI signs and symptoms, drug compliance, and the importance of partner management. Clients were also informed about the availability of 
RTI services in the clinic.

In SA1 first-time visits for FP/MCH clients took an average of twenty-two minutes, while follow-up/return visits took about fourteen minutes on average. This did not change much in SA2, with an average of twenty-four minutes and seventeen minutes, respectively.

\section{Client Satisfaction}

Most of the clients said they received the service they wanted and found the length of consultation "about right" and the providers' explanation "easy to understand". They also claimed there was privacy in the examination room. Clinic hours were found by clients to be convenient and waiting time reasonable (Figure 2).

\section{Figure 2. -- Clients' perception of length of waiting time}

( Experimental $\mathrm{N}=40$; Control $\mathrm{N}=13$ )

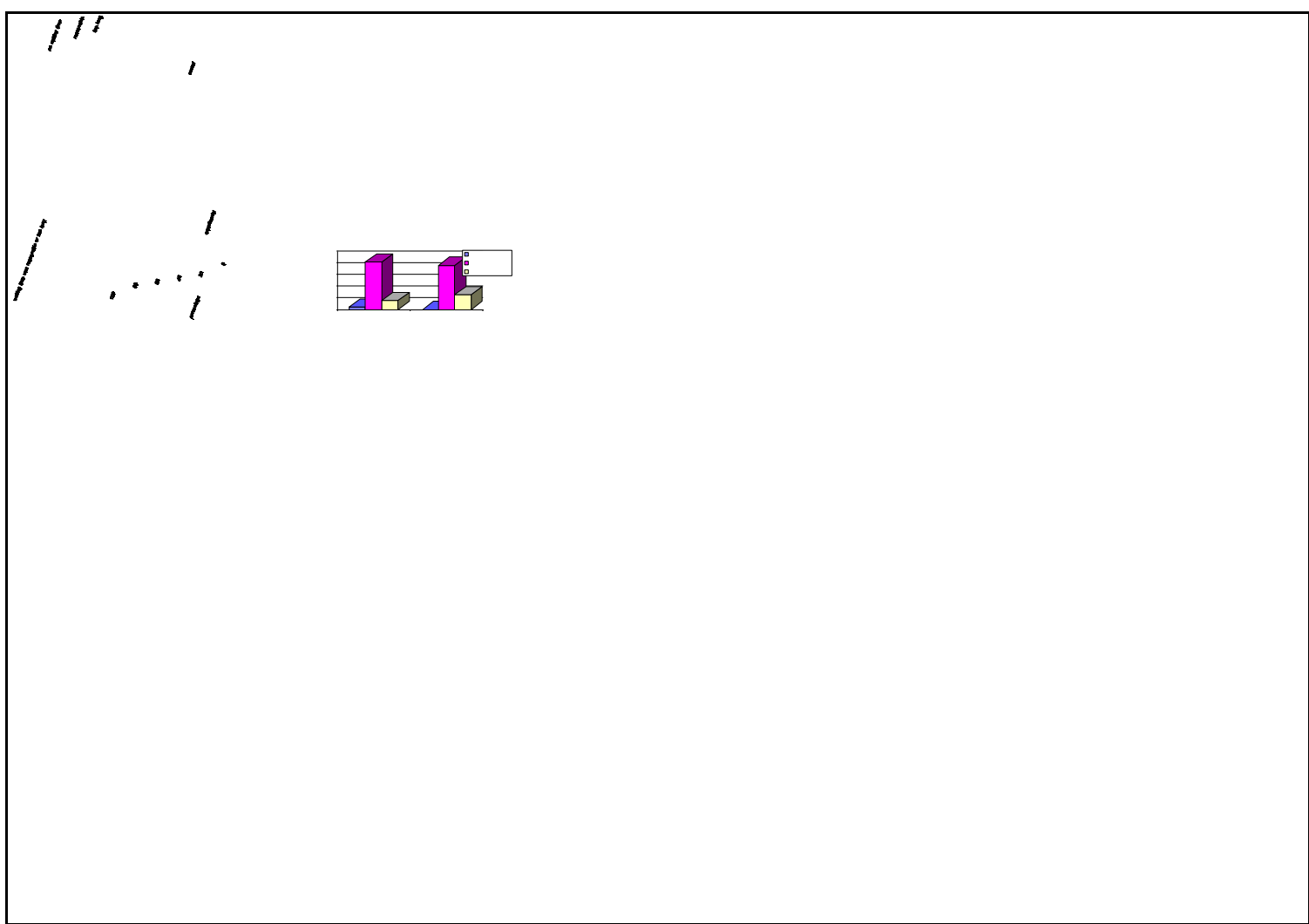


Client's perception of length of consultation was considered "about right". SA2 data showed that clients from the experimental clinics exhibit greater satisfaction on this variable compared to the clients from the control clinics (Figure 3). Figure 4 shows data about perception of clients regarding privacy during consultation. The figures here show that almost a hundred percent of clients from the experimental areas claimed enough privacy during consultation. Less than $80 \%$ claimed this to be the situation among clients from control clinics.

The majority of women (in both SA1 and SA2) preferred "accommodating" and "approachable" health providers, preferably of their own sex. Women in SA1 noted the "kind and caring staff" as the reason for liking their visit while women in SA2 noted the "competent staff" and "free medicines".

Travel time from home to the clinic usually took between fifteen minutes to an hour. All services were offered to the clients free of charge, although they were able to donate some small amount if they wished. 
Figure 3.--Client's perception of Length of Consultation Time

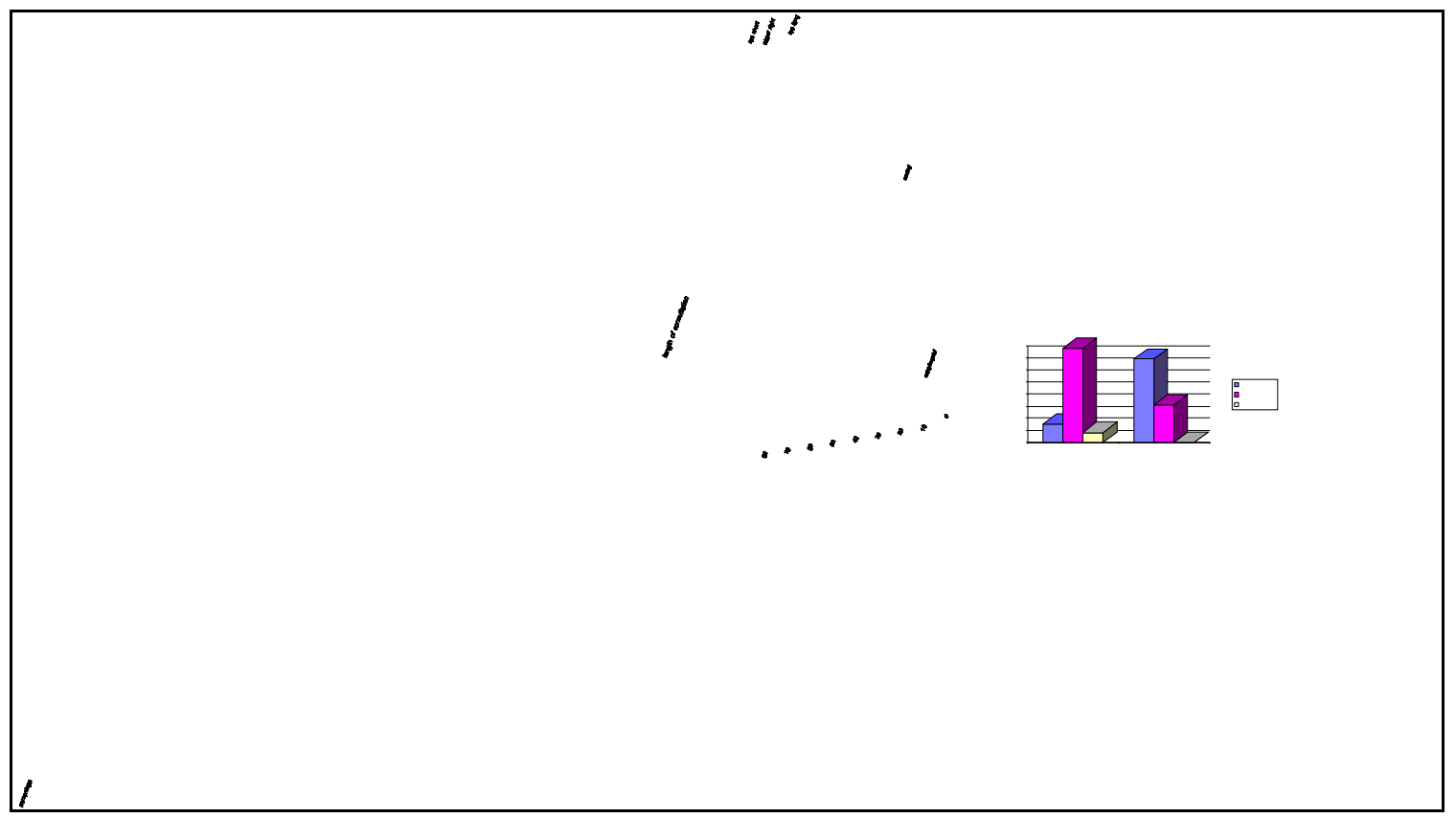

Figure 4.--Client Perception Whether There Was Enough Privacy at the Clinic during Consultation (percentage)

(Experimental $\mathrm{N}=40$; Control $\mathrm{N}=13$ ) 


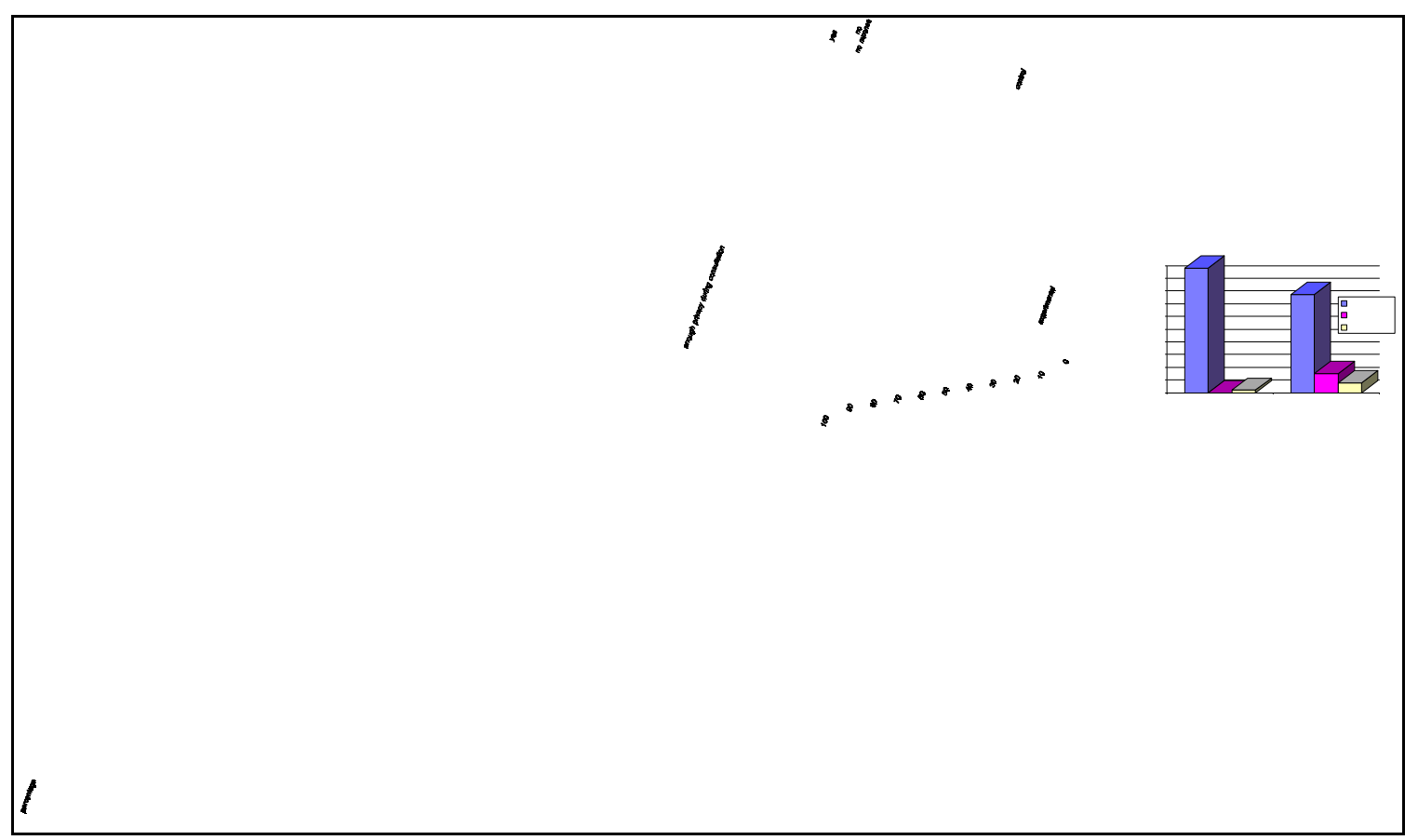

Most of the women had heard about STDs/RTIs, mostly from mass media like the newspaper, radio and TV. More women in the study clinics (31\%) were taught how to recognize RTI symptoms compared to the control sites where none of the women were educated about these symptoms.

In general, the results of the pre- and post-intervention situation analyses show that clinic facilities, staff capability, and client satisfaction substantially improved after the RTI intervention activities. RTI services in the seven experimental study clinics were previously either lacking altogether or were being addressed in a very inadequate fashion. After the six months' observation period, these seven clinics were found to be able to manage RTI cases with improved staff capability and adequate equipment and supplies.

Figure 5. -- Proportion of clients according to whether they were advised by the service provider on RTI prevention and treatment 


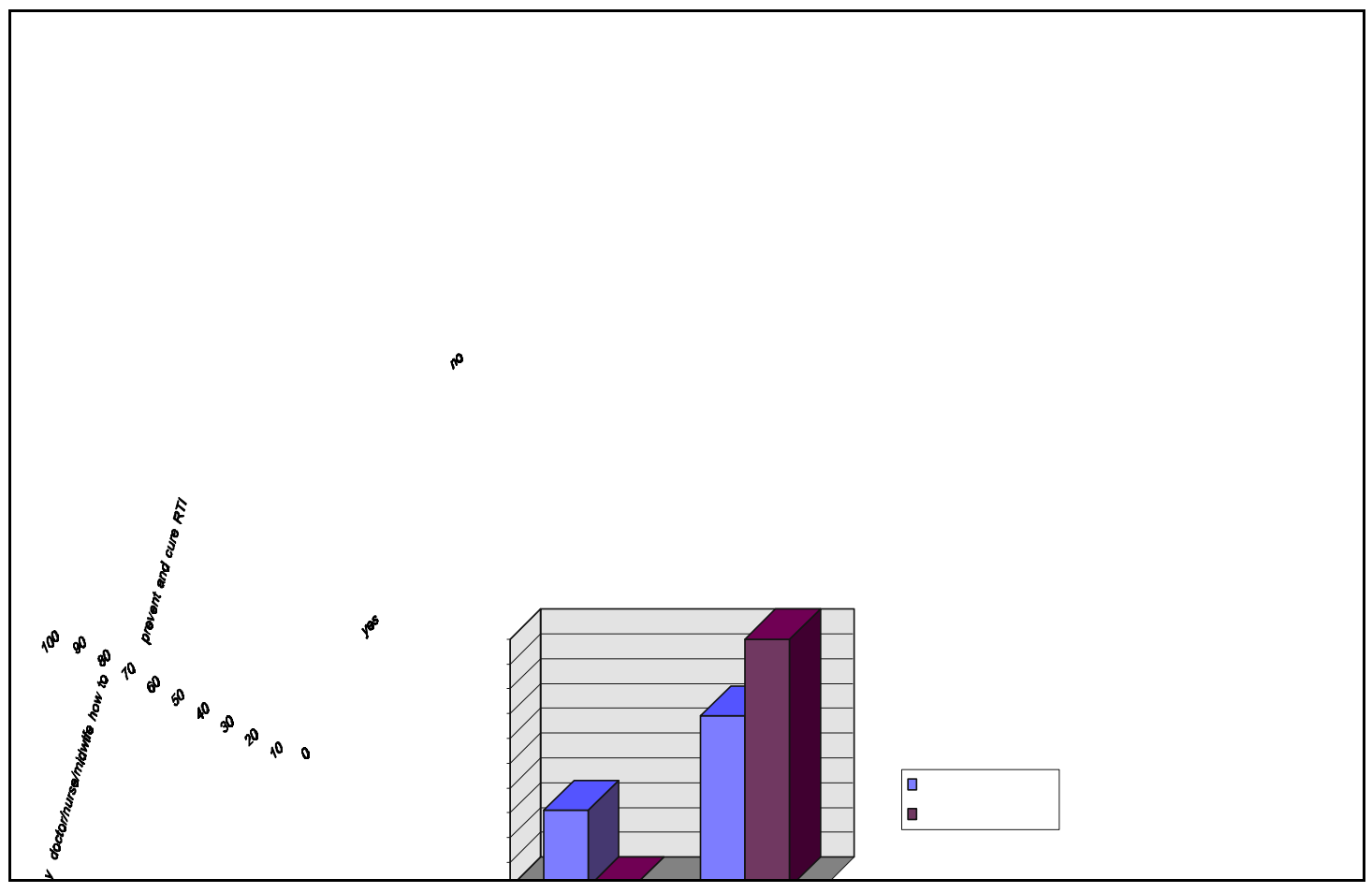




\section{RTI Case Management}

A total of 243 RTI cases were found in the seven health centers during the six-month observation period, from May to October 1997 (Figure 6). The RTI caseload was heaviest during the second half of the observation period. This trend was influenced mainly by three factors: (1) a system for drug provision was already in place during the second half of the study period; (2) more members of the community became aware of the availability of RTI services in the health center; and (3) providers became more consistent in asking FP/MCH clients about RTI symptoms as they grew more accustomed to the RTI forms.

Bernardo showed the highest number of RTI cases diagnosed. The three study clinics in Cagayan de Oro showed that the integration process was derailed by the overload of clients in these SDPs and the inability of a physician to visit the clinics on a daily basis.

Figure 6. --RTI Cases Identified in Study Sites, May-October 1997 (Percentage)

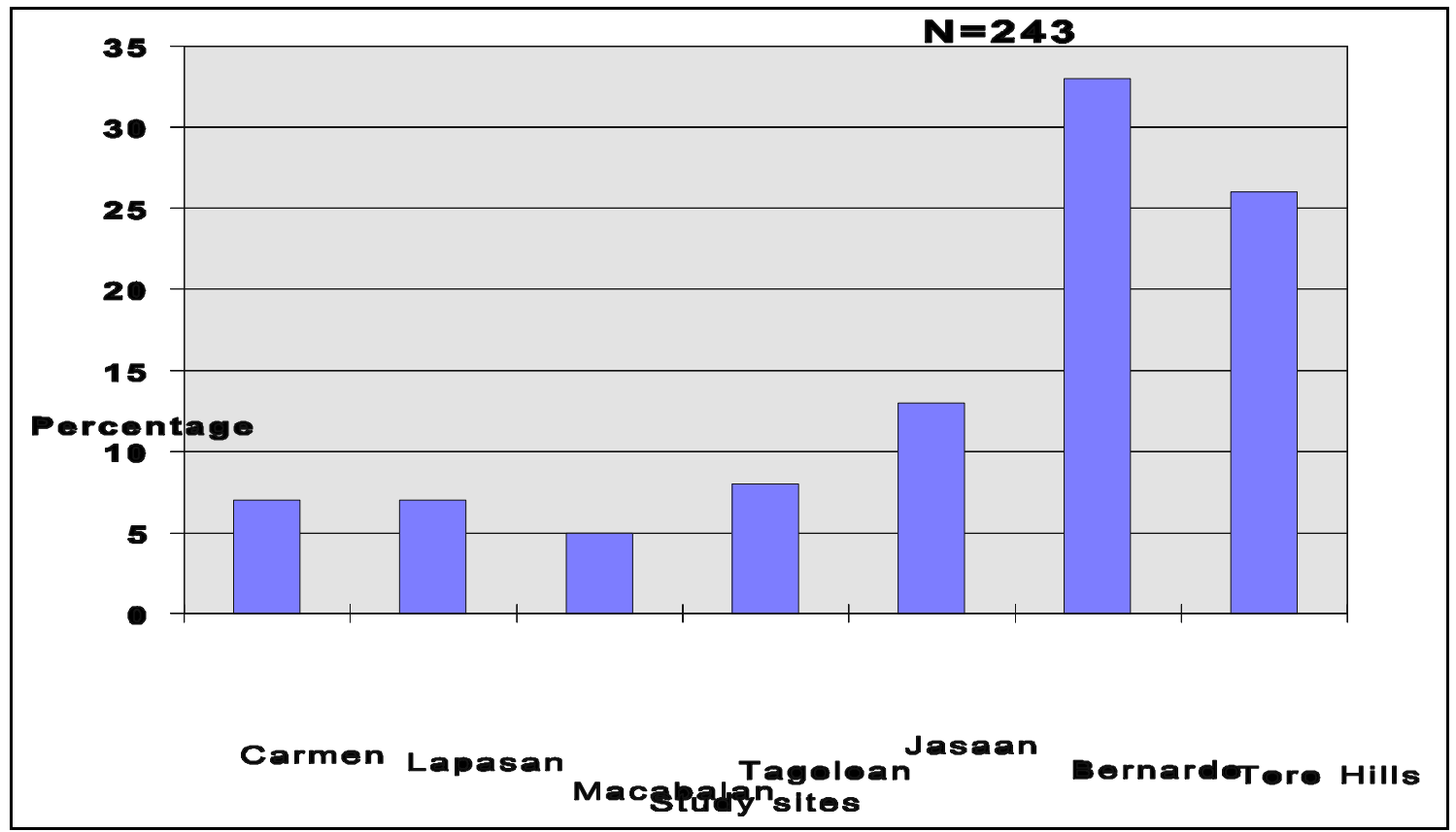

In order to look at the extent of the problem of RTIs, among FP clients data from service statistics, data on RTI among new FP acceptors were collected in the three clinics mentioned earlier. This yielded an RTI prevalence of $8 \%$ among new FP acceptors (see Table 2). 
Table 2.-- Percentage of New FP Acceptors with RTIs in Selected Clinics Where FP Clients Are Screened of RTI Symptoms, May-October 1997

\begin{tabular}{|l|c|c|c|}
\hline Health Center & No. of RTI Cases & $\begin{array}{c}\text { No. of New FP } \\
\text { Acceptors }\end{array}$ & $\begin{array}{c}\text { \% of New FP } \\
\text { Acceptors w/ RTI }\end{array}$ \\
\hline Jasaan & 5 & 45 & 11 \\
\hline Toro Hills & 2 & 212 & 12 \\
\hline Bernardo & 42 & 366 & 8 \\
\hline Total & 49 & 634 & 12 \\
\hline
\end{tabular}

The findings in these three clinics may be compared to the 1994 data (Table 2.1) found among women attending antenatal clinics at the Philippine General Hospital conducted by AIDSCAP and the University of Washington.

Table 2.1-- STI/RTI Prevalence in Low Risk Population in Women Attending the Antenatal Clinic at the Philippine General Hospital, July-September 1994

\begin{tabular}{|l|rr|c|}
\hline \multicolumn{1}{|c|}{ Etiology } & \multicolumn{2}{|c|}{ Philippines $^{\mathrm{a}}$} & Vietnam $^{\mathrm{b}}$ \\
\hline Gonorrhea & $1.0 \%$ & $(2 / 203)$ & .2 \\
Chlamydia & $5.6 \%$ & $(10 / 178)$ & .8 \\
Syphilis & $1.0 \%$ & $(2 / 203)$ & 1.0 \\
Trichomoniasis & $0.5 \%$ & $(1 / 203)$ & 3.1 \\
Bacterial Vaginosis & $11.3 \%$ & $(23 / 203$ & 6.7 \\
\hline
\end{tabular}

a Adopted from Renee Faldas, "Overview of Prevalence, Diagnosis and Treatment of Chlamydia and Gonorrhea in the Philippines," paper presented at the International Workshop, Bogor, Indonesia, 4-5 December 1997.

b adopted from Chris Elias , "The Prevalence of Reproductive Tract Infection," paper presented at the International Workshop, Bogor, 
Indonesia, 4-5 December 1997.

a) Profile of RTI Clients

Eighty-six percent of the 243 RTI clients were female. This heavy proportion of female RTI clients is explained by the fact that women make up the captive group of FP/MCH clients.

The age of RTI clients ranged from two weeks old (one case of an infant with neonatal conjunctivitis) to 65 years of age. Most patients, however, fell within the reproductive ages. The average RTI client was 31 years old, married, and was either a housewife or a clerk/employee. The majority of their partners were construction/factory workers, drivers, or clerks/employees. RTI clients had an average of 2.8 children.

Figure 7.-- Percent of RTI clients by Sex 


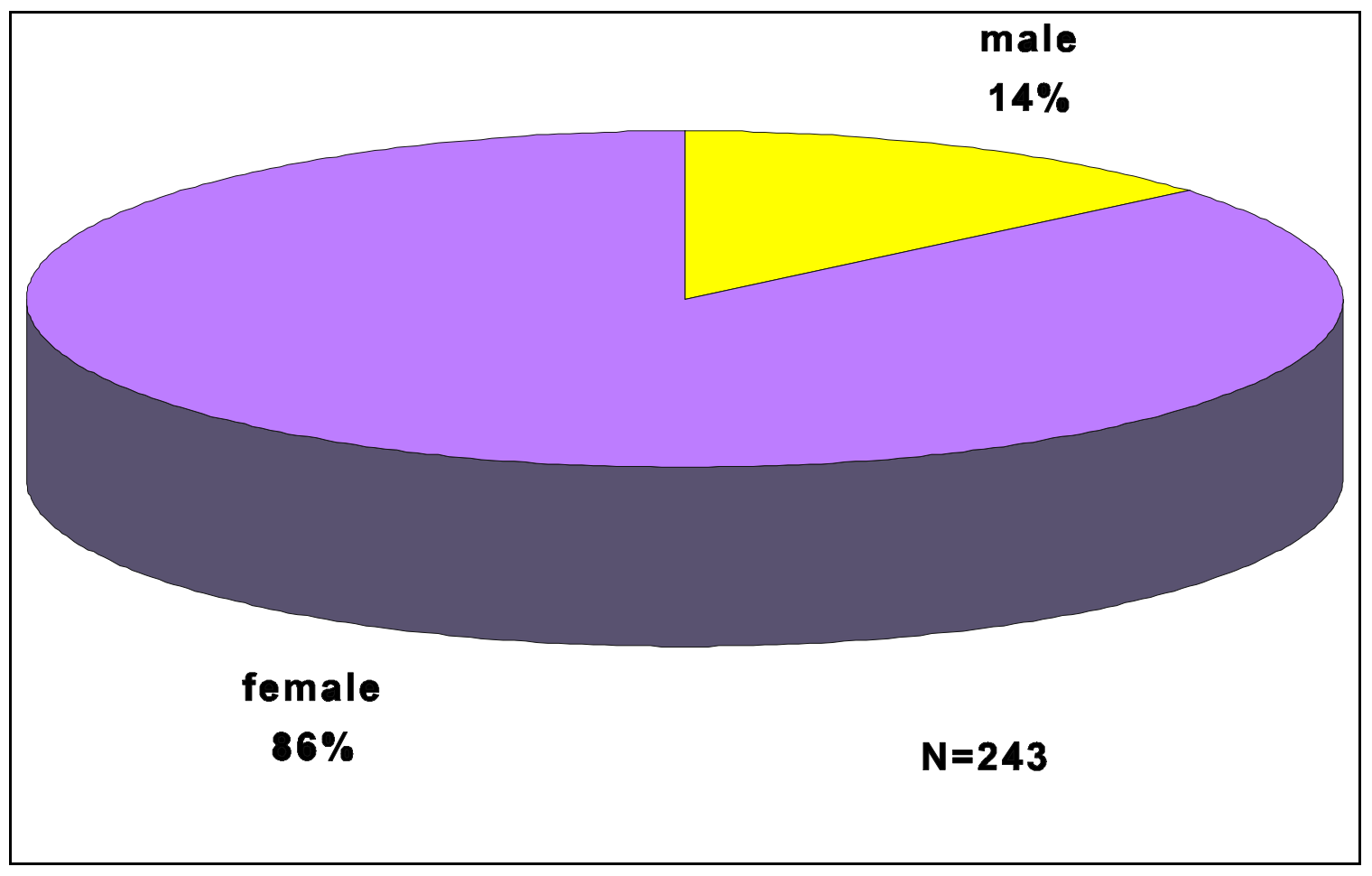

Figure 8. -- Percent of RTI Clients by Marital Status 


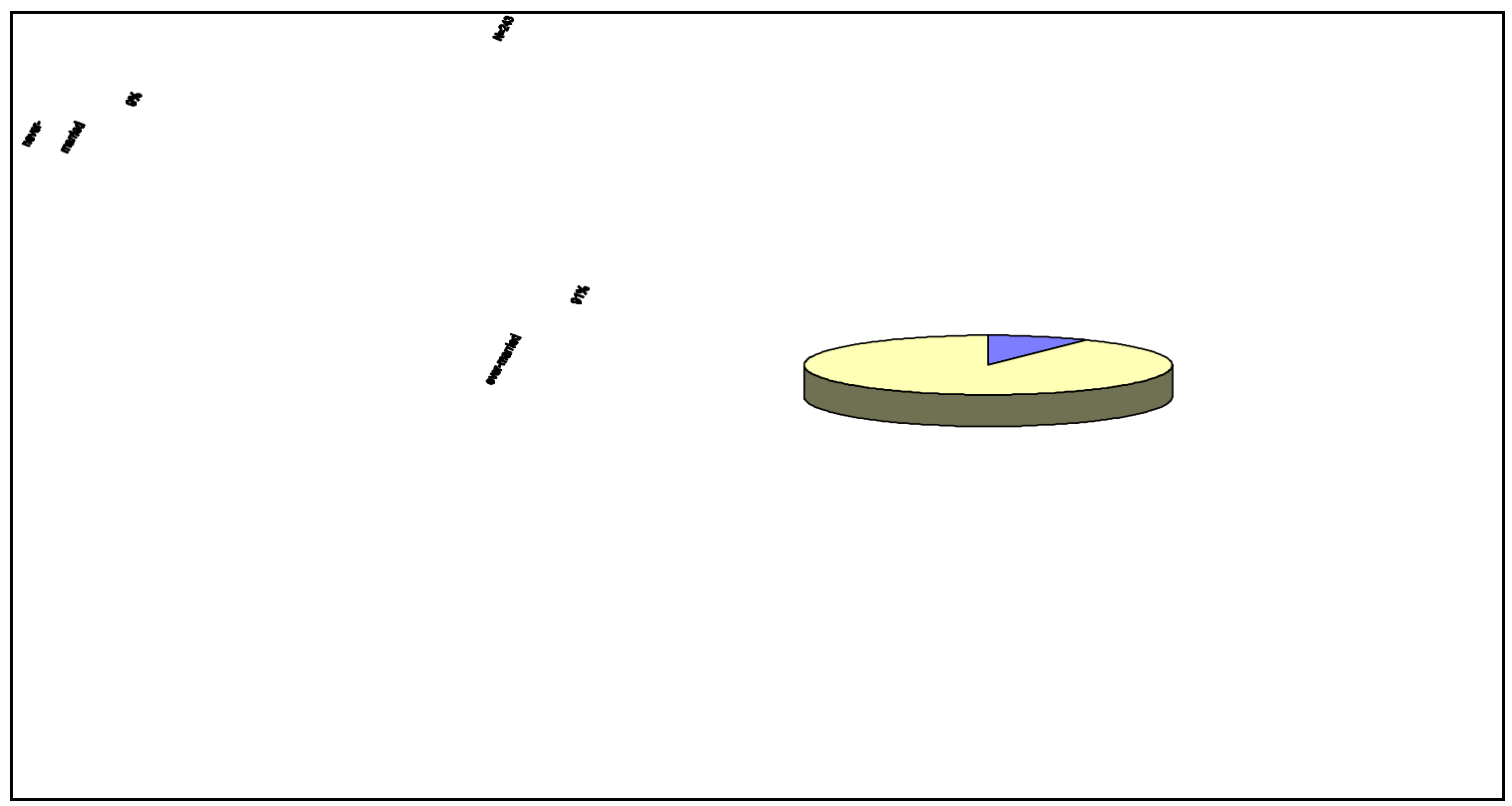

Figure 9.--RTI clients by Occupation Study Sites, May-October 1997

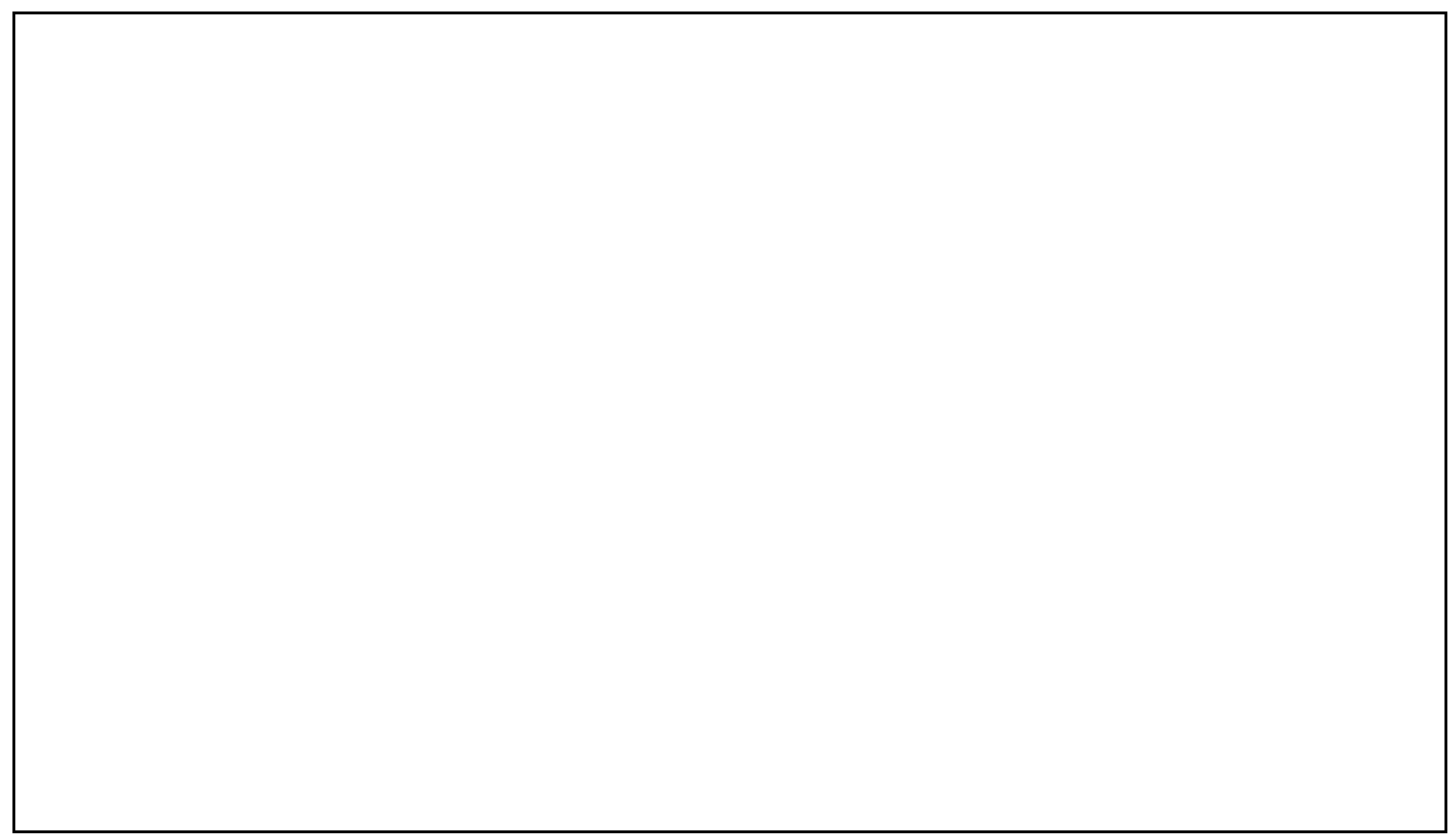

Figure 10.--RTI clients by number of children, May-October 1997 


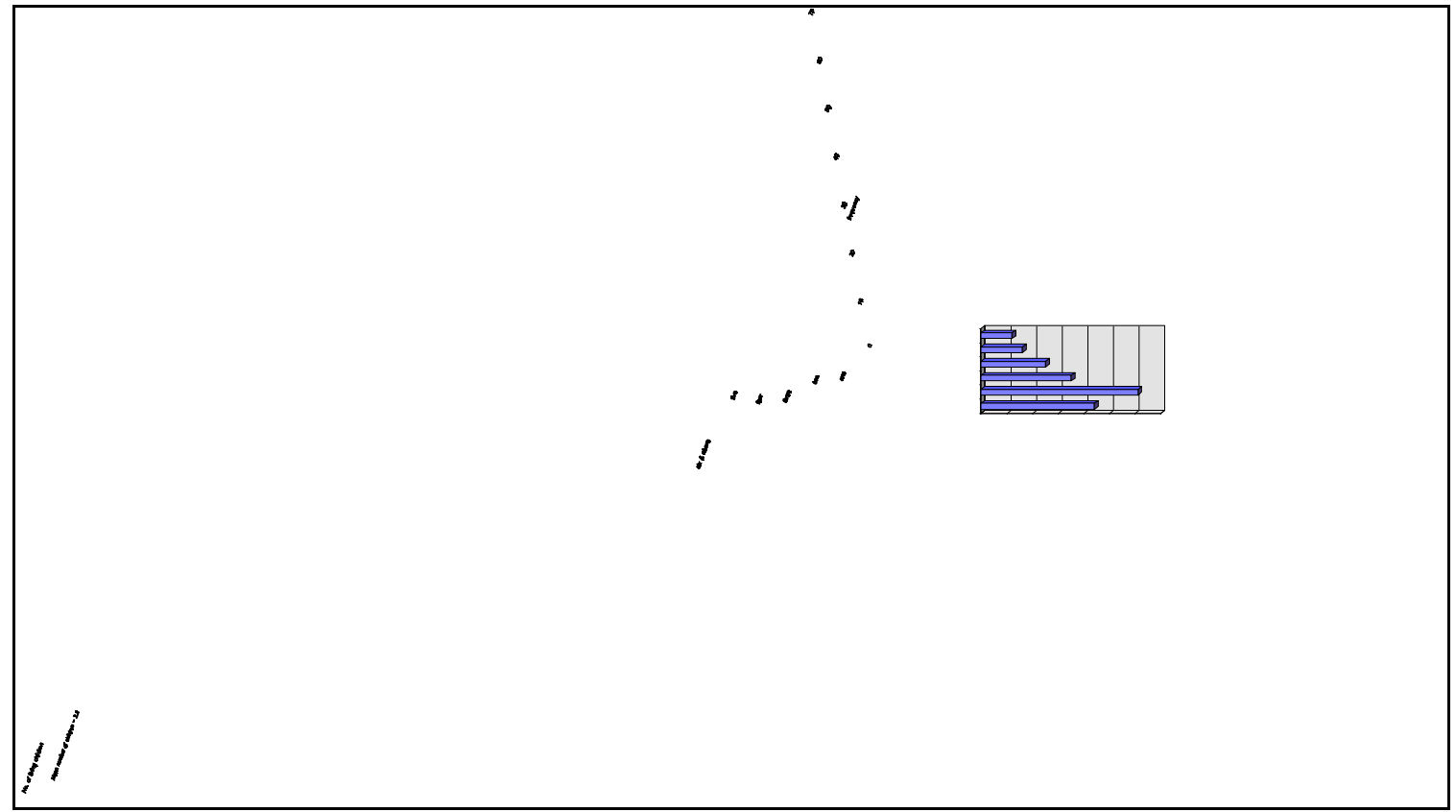

b) Method of Casefinding

More than half of the female clients were walk-in clients who came with complaints to the service provider (Figure 11). About 20 percent of the asymptomatic RTI cases were discovered as incidental findings; that is, during either one of the following: annual check-up for continuing users; Pap smear; IUD check-up (during the first six months); physical exam for new FP acceptors; and speculum exam prior to IUD insertion (Figure 12). Most of these incidental findings were vaginitis cases, which were observed to be usually negative in the risk assessment.

Almost one-fifth of the female RTI cases were referred from other SDPs, particularly from other health centers within the vicinity and from barangay health stations (BHSs), whose midwives were also trained in syndromic management as part of the study intervention. This large portion of referred cases was also due to the persistent efforts of physicians to ask for referrals from BHS midwives and from other health center physicians in the same district. About four percent of the cases were discovered during prenatal exam, while two percent of the females were partners of male clients with RTI, cases that were found through contact tracing. 
Figure 11. -- Method of RTI Case Finding, Female Clients, May-October 1997

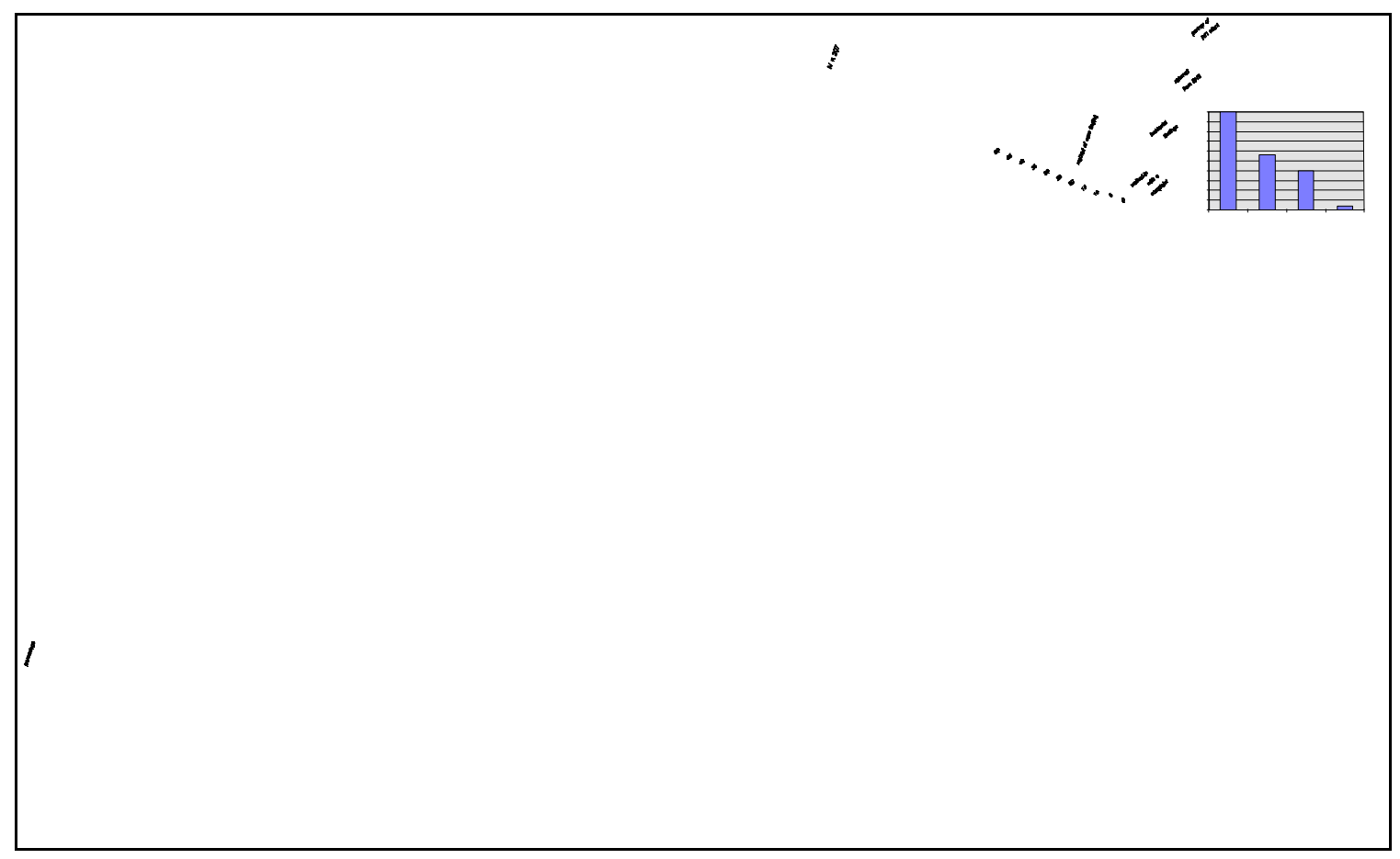

Figure 12. -- RTI clients by incidental findings 
While only a small proportion of the female RTI clients were found through contact tracing, more than half of the male RTI clients were partners of clients with RTI (Figure 13). A large proportion of the men, about 35 percent, walked in with a complaint. Only less than one percent were referred from other SDPs or BHSs.

Figure 13. -- RTI clients by method of case finding for Male clients 


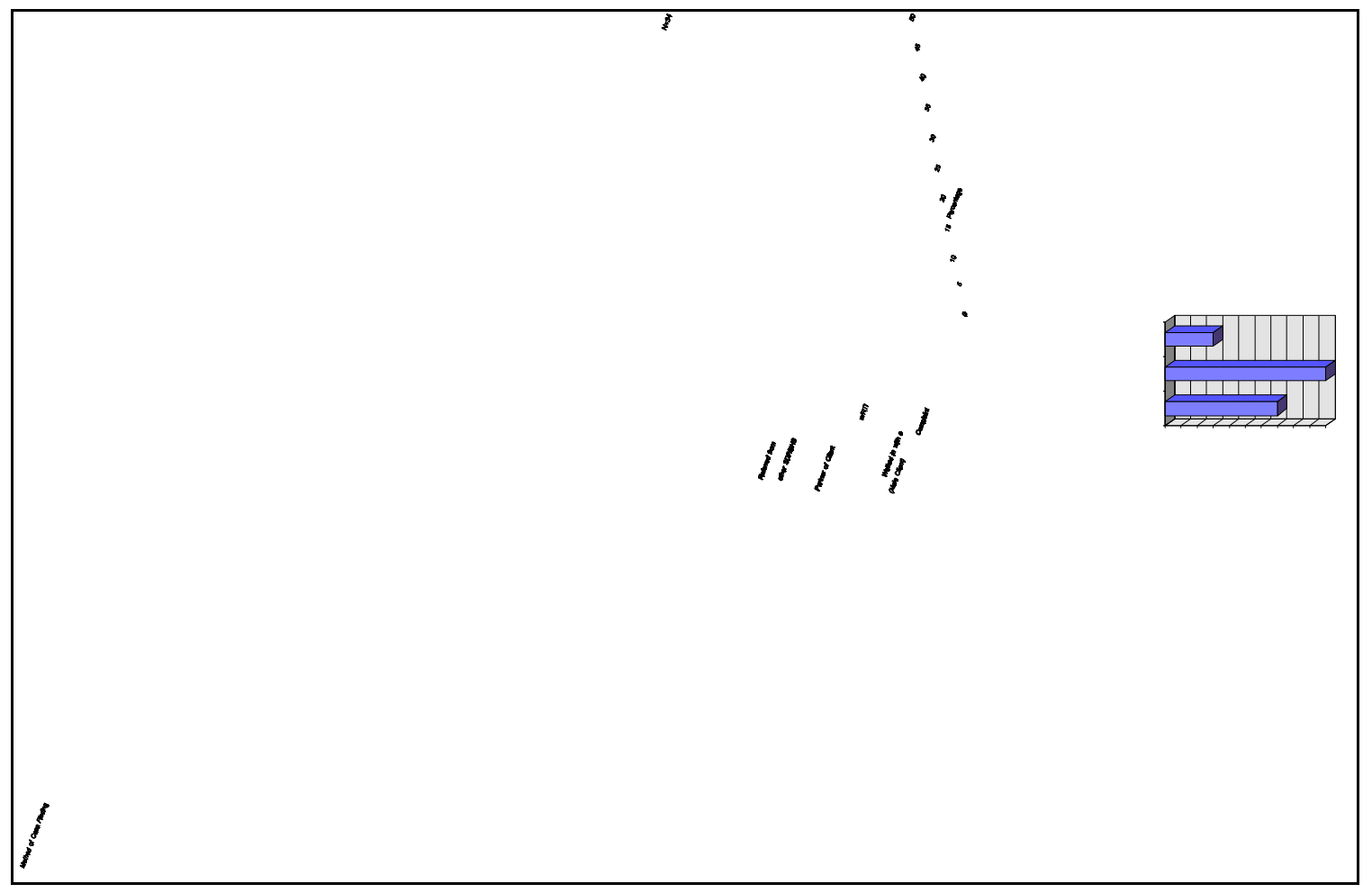

c) Chief Complaint

Sixty percent complained of abnormal vaginal discharge. Close to 14 percent complained of genital itchiness, while nine percent suffered from lower abdominal pain (Table 14). Other related complaints for the women were pain on urination and pain during sexual intercourse. Among men only, the chief complaints were urethral discharge (34\%) and painful urination (25\%). Thirty one percent of the male RTI clients were asymptomatic but were identified through partner notification and were treated as RTI clients.

Figure 14.--RTI Clients by Chief Complaint, All Clients, May-December 1997 


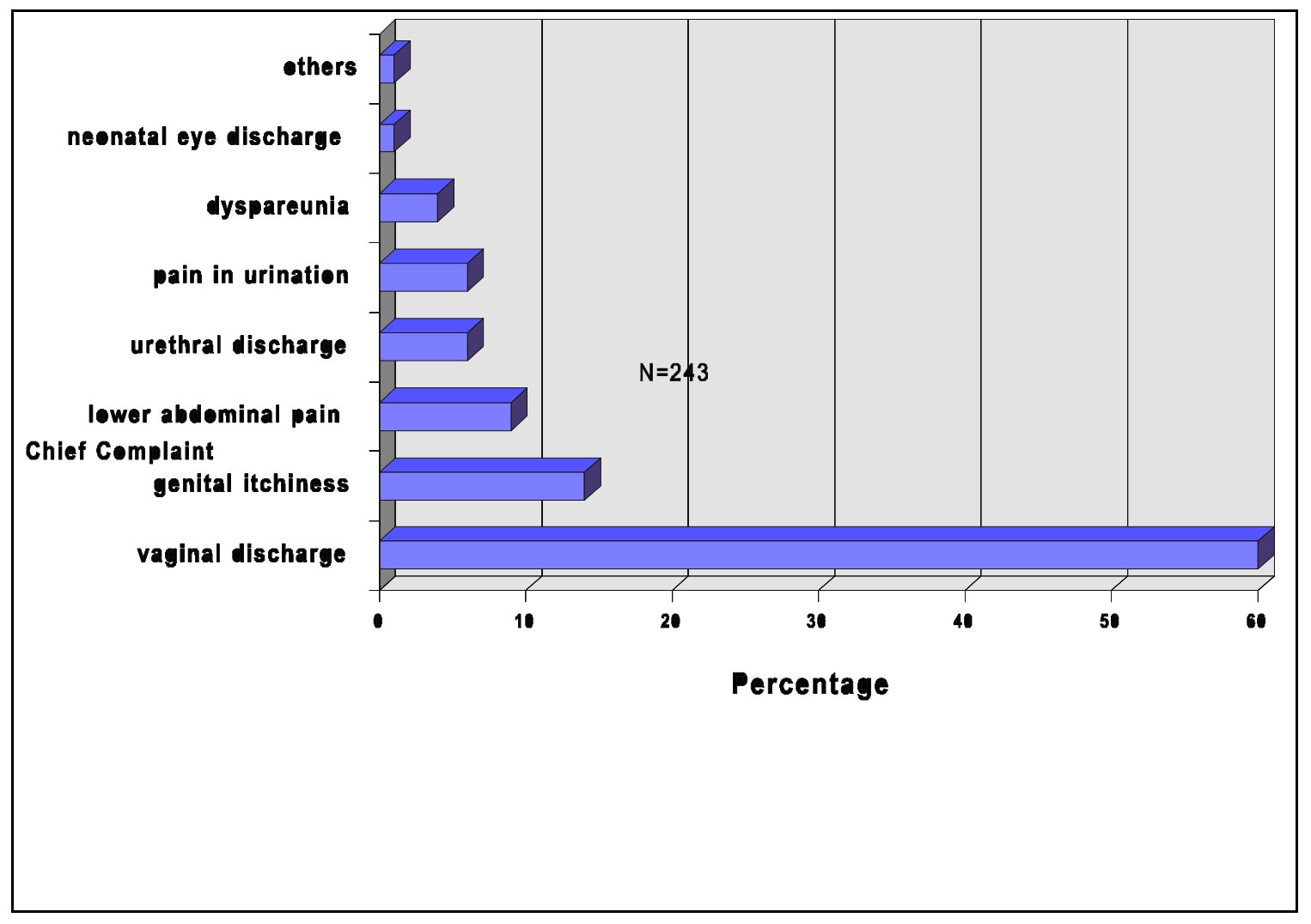

Figure 15.--RTI clients by presenting symptom (percentage) 


\section{d) Risk Assessment Questions}

The risk assessment (RA) procedure used a set of questions to distinguish vaginitis from cervicitis in women with vaginal discharge. Since the risk assessment (RA) questions were incorporated in the RTI individual treatment records, the providers were able to gauge the risk of RTI transmission as added information for arriving at a diagnosis.

Thirteen percent of the RTI clients, for instance, believed that their partner may have had other (another) sex partner in the last two months. Another nine percent believed their partner had an STD or had been treated for an STD. Seven percent admitted having had more than one sex partner, while two percent changed sex partners, in the last three months.

Some clients were not completely certain about their spouse's activities and would answer "maybe" or "it seems so" to the RA questions. The providers in the study considered cases like these positive for RTI risk, although they felt that there was a need to differentiate between ambiguous answers such as "hindi ko alam" (I don't know) and "suspetsa ko lang" (It is only my suspicion). In addition, the knowledge of the health worker about the personal background of the client or her partner could also influence the RA results. For instance, at least one provider admitted viewing a client as positive for RTI risk because the provider had personal knowledge about the (sexual) activities of the client's spouse, even though the client said "no" to all the RA questions.

The providers reported no major problems encountered in asking the RA questions, particularly for the walk-in clients, although they observed that the questions were rather personal and direct. Extra effort had to be given, though, in asking the same questions of clients whose infection was incidentally found during prenatal and FP exams. Physicians had to be extra careful in explaining the need for accurate answers during risk assessment, and the need for a physical exam for a correct diagnosis. Nonetheless they assured clients that whatever personal information they gave would be kept confidential.

The reactions of the clients to the RA questions varied from "embarrassment, discomfort, and unwillingness to respond to the questions" to "openness, amusement, and laughter." 
e) Current FP Method

Fifty-nine percent of the RTI clients were non-family planning users. Of the remaining 51 percent, twenty-three percent (29) were IUD users and fifteen percent (19) were pill users. Six percent (7) used DMPA. Only two percent of the RTI clients claimed they were condom users. This finding illustrates the need for providers to explain the importance and correctness of condom use, especially among RTI clients. If this is done, it might improve the use of condoms among couples. Despite the rather liberal distribution of condom among RTI clients, it seems like this has not been used for FP. Their use in this context may have been simply for prevention of RTIs.

Fig. 16. -- Current FP Method Used by RTI Clients, May-October 1997

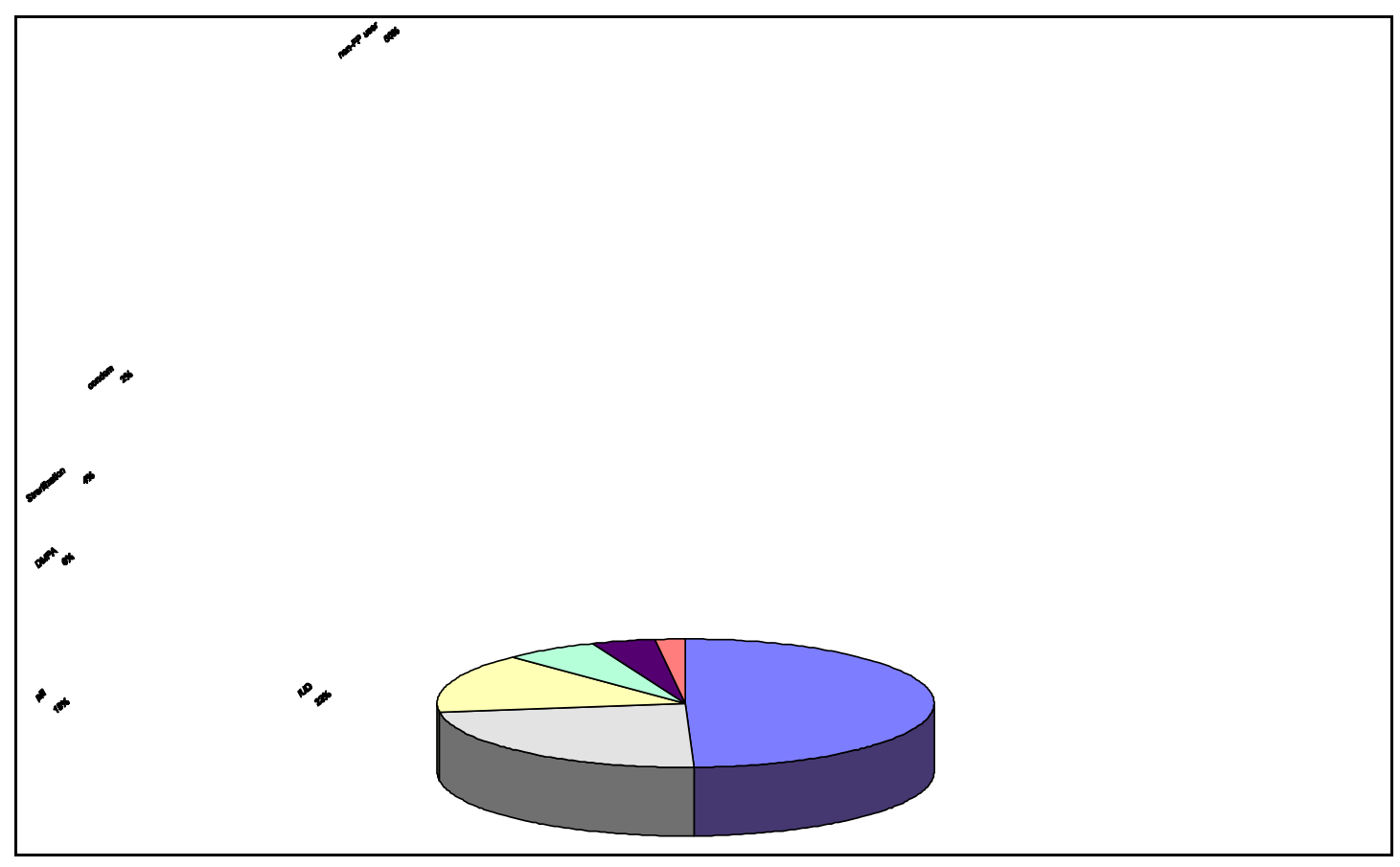

Although conclusions about the correlation of FP use (or for use of particular methods) and RTI prevalence cannot conclusively be made in the present study, the need to determine this through further research and analysis is evident as this could affect demand for FP services when RTIs are perceived as side effects of FP use. 
f) Laboratory Tests

Four of the seven health centers in the study were equipped with laboratory facilities with which simple microscopy and lab tests could be done. Tests to determine RTI (KOH, NSS, Gram stain) were done in 31 percent of all RTI cases. Pap smear was taken in $18 \%$ of the cases, but the readings were done mostly in private laboratories. Urinalysis was performed in $6 \%$ of the total cases, mainly to rule out UTI for the diagnosis (Table 16).

These lab tests were done by a trained medical technologist in the health center. The results were used by the doctor to corroborate his/her initial diagnosis. In cases where the lab test results did not concur with the results of the physical exam and the risk assessment, the providers reported relying more on the clinical presentations of the client and their own clinical experience and knowledge rather than on the laboratory results. When lab tests did support their presumptive diagnosis, the providers reported feeling an increased confidence in managing the client.

One doctor claimed that risk assessment was "very helpful" in her determination of vaginitis as opposed to cervicitis. Another doctor felt that "even a positive risk assessment should always be followed by a proper physical examination, because your observations during a physical examination will boost confidence in your initial findings."

The overall opinion of the doctors seemed to be that, even though clients were initially embarrassed by some of the RA questions, they were generally willing to give honest answers "because they fear the consequences if they keep information from the doctor." The providers also reported that valid answers could be elicited when the provider used proper strategies in interviewing clients. It is important to make the client feel at ease and remind them of the importance of complete, factual information so that they can be properly treated.

One doctor felt that the accuracy of the RA could be improved by adding a few questions. For instance, these could include questions on the occupation of the spouse and 
whether or not the spouse had been away for some time (i.e., those working overseas).

While syndromic management does not require laboratory tests, one doctor said, "There are instances when you need to do these tests." She enumerated several instances where laboratory tests can help diagnosis: (1) resistant cases which do not improve with antibiotics, (2)recurrent RTIs, and, (3) children with symptoms suggestive of RTIs (particularly in very young girls with abnormal vaginal discharge).

g) Diagnosis and Management

Close to sixty percent of the clients were diagnosed with vaginitis/cervicitis. Seventeen percent had trichomoniasis/candidiasis while 10 percent were diagnosed with gonorrhea/chlamydia. Almost five percent had bacterial vaginosis and three percent had PID. Mixed infections were also noted. Moreover, in $4 \%$ of the RTI cases, UTI was diagnosed as well. The provider arrived at these diagnoses mainly through syndromic management $(75 \%)$. The rest of the diagnoses were based on the client's medical history, physical exam, and laboratory tests $(12 \%)$, and on history and physical exam only $(8 \%)$.

Figure 17.--Percentage of laboratory exams conducted by type of exam 


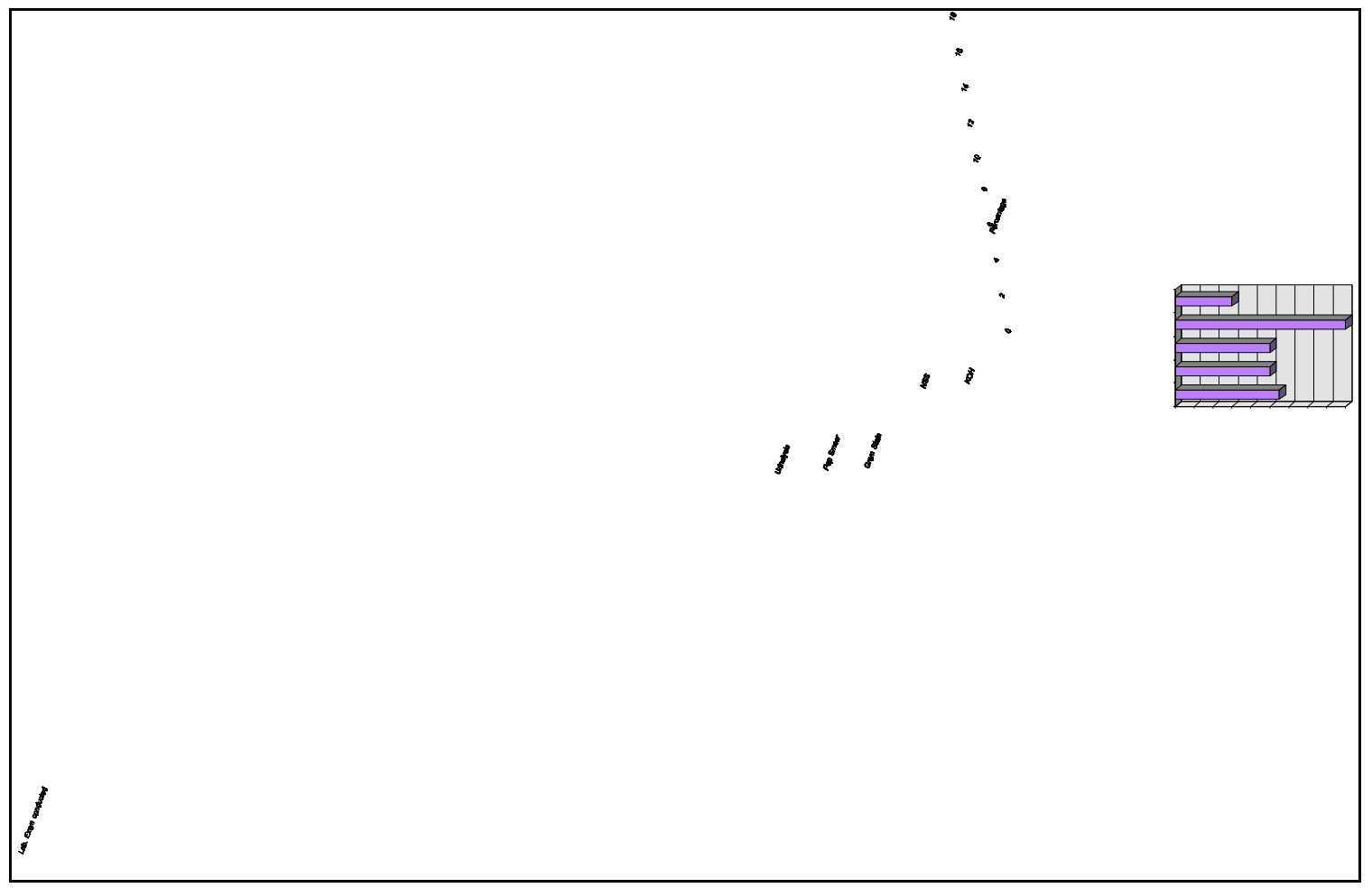

RTI records completed by the physician showed that 98 percent of the clients were given drugs for treatment and instructions regarding how these should be used, with regard to following the suggested RTI protocols. It was observed that 97 percent of the clients were given counseling and health education messages. Ninety-five percent of the clients were also scheduled for a follow-up visit. In this sense it seems as though the integration of RTI services have proceeded quite well. However, there is still room for improvement. The proportion of clients given information to encourage condom use was lower than 89 percent. Furthermore, providers seemed not to be consistent in giving messages or information to encourage treatment of the client's sex partner. For example, only 81 percent of these cases were encouraged to bring their partner for treatment.

In one urban clinic, the doctor scheduled consultations in the afternoon, when client load was considerably lighter. This was done so that there would be enough space and privacy for PE and counseling. She considered this a strategy to make sure that adding the additional 
health service did not jeopardize the other services that were already in place, and at the same time the demands for privacy and space were also met.

In the course of the study, at least two physicians reported finding the management of a few RTI cases a bit problematic. These cases involved management of pregnant clients with RTI and a three-year old child having abnormal vaginal discharge. The doctors felt they needed additional guidance in the management of these special cases, especially since the existing guidelines lack specific instructions on these particular cases.

\section{h) Drug Provision}

The majority of the patients $(75 \%)$ received a complete amount of drugs, while eight percent were given a partial amount of drugs, (whatever medicines that were available in the clinic). About $15 \%$ of the clients were given drug prescriptions, particularly during the first month of the study when regular drug provision was not yet installed (Figure 17). The doctors observed that clients who were given drug prescriptions showed poor compliance, delayed revisits or no revisits at all.

Drug provision received a boost in the rural health unit in Jasaan when the municipal health officer was able to convince the Mayor to include P50,000 $(\$ 1,250)$ in the 1998 municipal budget for the purchase of RTI drugs. The physician hopes to sustain drug provision even after the end of the RTI project. In Quezon City, the City Health Office has continued post-project RTI drug provision to Bernardo and Toro Hills Health Centers. This was made possible through budget allocations in the city government and through drug funding support from the Urban Health and Nutrition Program.

Even during the early inception of the project, the study team visited the local government officials (mayors, provincial planning officers and municipal health officials) on a number of occasions to solicit their support for the program. The cultivation of this rapport with the local officials emphasize the importance of cooperation of the LGUs. 
Fig. 18. -- Drug provision for RTI clients

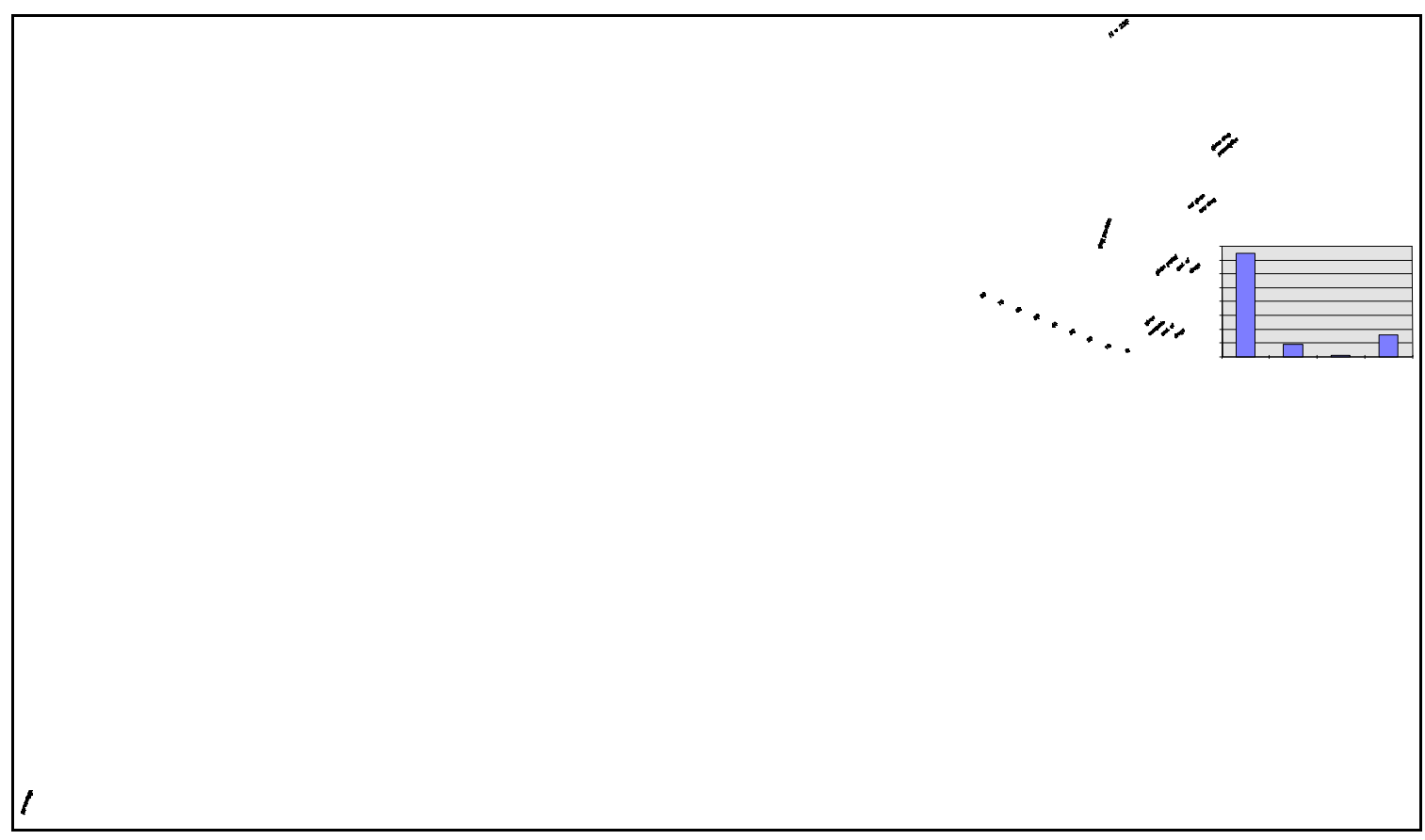

i) Follow-up Visits

Clinic data analyzed by physicians who were part of the study showed that a high proportion of their clients did return for a follow-up visit. About $74 \%$ of RTI clients returned for a revisit, of which $66 \%$ showed clinical improvement. Many of those with no clinical improvement during the first visit returned for a second visit (14\%) and a third visit (3\%). This finding on return visits is encouraging considering the general observation that patients rarely come back to the clinic for follow-up visits. The physicians attributed this high rate of return visits to their message which emphasizes the need to follow doctors' instructions for the client to be completely cured. They also reported that BHW's and midwives's consistent follow-ups and reminders of clients' scheduled appointments, also increased the 
return rate. It was also observed that return visits increased simultaneously with the availability of RTI drugs in the health center. The availability of "free drugs" also helped to attract clients to come back to the health center.

Follow-up interviews with clients identified various reasons for patients who did not return for a check-up. These included the following: (1) they were relieved of their symptoms; (2) the health worker failed to tell them to return; (3) they were dissatisfied with the services received or the health workers were too busy to give them attention when they visited the clinic; (4) they were not able to buy the prescribed drugs, and therefore they hesitated to go back to the health center.

\section{j) Partner Notification}

Service providers in the seven health centers reported that they encouraged RTI clients to inform their sex partners about the results of their consultation. More than eighty percent of all clients were told to bring their partners to the health center. Symptomatic partners who came were treated and counseled; asymptomatic partners were counseled to use condoms during the period of treatment of their partners or to avoid sexual intercourse until the partner was cured.

Women patients were particularly concerned about RTI transmission and would sometimes volunteer for sexual abstinence while on treatment. Some husbands unfortunately refused examination and possible treatment, citing the following reasons: (a) I don't need treatment; anyway we haven't had sex for a long time"; (b) "I don't feel anything, I'm not sick"; (c) My wife is the one who is sick, not I." These prevailing attitudes hampered partner management, and consequently, some providers reported that they were not always successful in their efforts at partner management.

k) Referrals to Other SDPs 
Four RTI clients from Cagayan de Oro City were referred to a higher level SDP, notably the Northern Mindanao Medical Center, to treat the following cases: an RTI with recurrent UTI; RTI resistant to Miconazole; cervical polyp; and severe Bartholinitis. The rest of the RTI cases were managed at the seven health centers.

1) Post-Intervention Developments: Service Delivery, Dissemination and Utilization

The study team visited the study sites and found that RTI cases are still being managed in the study clinics six months after the end of the project. In Quezon City, RTI drugs in Bernardo and Toro Hills Health Centers continue to be provided by the City Health Office. Referrals from other health centers have increased since October 1997. The same has happened in Cagayan de Oro City. In fact, the four study clinics in the city have been designated by the City Health Office as referral centers for RTI cases. Quezon City health officials are considering similar plans: they intend to have at least one more RTI referral center in each of the four districts in Quezon City, in addition to Toro Hills and Bernardo.

The Population Council co-sponsored a research dissemination workshop in Cebu City with the National Task Force for Social Science and Reproductive Health in January 15-16, 1998 featuring the results of this study. The workshop was attended by policymakers and program managers, donors and the academe. Another conference was held in Manila on May 26, 1998, where four projects were presented, among them was the results of the RTI Study. The dissemination conference fulfilled the objectives of sharing the results of the study and stimulating links and networking with other RTI initiatives in the country to address the issue of provision of RTI Services for prevention and diagnosis.

\section{COST ANALYSIS}

The project also investigated the financial implications of a nationwide integration of RTI services within the local public health clinics to address sustainability and replication issues. A cost analysis of adding RTI services within the Family Planning/Maternal and Child Health program is particularly important in the Philippine setting, where primary health care is currently being provided by devolved local government units (LGUs). Clearly, LGU officials are not going to support such a programmatic change without first being able to assess its 
financial implications.

\section{Objective}

The primary objective of the cost analysis was to provide cost models of standardized RTI management applicable to two types of clinics: one with laboratory facilities and one without laboratory facilities.

\section{Unit of Study}

Data were collected at the level of a centrally located health care center, known as a Rural Health Unit (RHU) for rural areas and an Urban Health Center for urban areas. Under each RHU are Barangay Health Stations, each of which is usually managed by a midwife. The RHU is typically staffed by a medical officer, a public health nurse, a public health midwife, and a clinic aide. Some clinics have a dentist and a medical technologist. The standard doctor-patient ratio in the public health system is supposed to be 1: 25,000, although in reality the client load is typically much larger.

\section{Methodology}

The production process methodology was used in the cost analysis. This approach describes how resources or inputs are converted by processes to outputs and outcomes (Janowitz and Bratt, 1994). The components of RTI service delivery were grouped as either inputs, processes, or outputs. Inputs include personnel, training, clinic and laboratory equipment and supplies, IEC materials, and drugs. These components combine to provide RTI service delivery in the health center. The expected outputs are clients who seek RTI services, RTI counseling and education (both one-on-one and community-wide), laboratory testing, and treatment.

The two models describe the marginal cost of RTI service delivery. Only additional resources needed to provide RTI services were costed. Building rent and salaries of personnel, for instance, were not included in the cost analysis. 
Costs were classified as being either fixed or variable. Fixed costs included didactic and practicum training of service providers, IEC activities, clinic equipment, and laboratory equipment. Variable costs included MIS forms, clinic supplies, laboratory tests, and RTI drug costs.

Costs were computed for the first year of integrating RTI services. Minimum and maximum costs were computed to allow for cost differences in certain items as travel allowances, sizes of clinic equipment, wholesale vs. retail prices, and type of drugs used.

\section{Sources of Data}

Data on training costs were obtained from the Economic Development Foundation (EDF), an NGO that the Department of Health has contracted to conduct all its health training programs. The costs of clinic equipment and supplies, laboratory equipment, reagents, RTI drugs, and IEC materials were based on prevailing market rates in Metro Manila and Cagayan de Oro City.

\section{(1) MODEL 1 : CLINIC WITHOUT LABORATORY}

Model 1 describes cost requirements in integrating RTI management services in a main health center that has no laboratory capacity. The clinic personnel use the syndromic management approach, which the Philippine Department of Health has endorsed for the management of RTI cases. Diagnosis and management make use of algorithms for specific syndromes.

Treatment costs varied according to the severity of infection and the type of drugs being used (e.g. whether first or second generation drugs, whether branded or generic, locally manufactured or imported). In this study, drugs were chosen according to the recommendations set by the National STD Management Guidelines of the Department of Health. The costs of treating the regular sex partners of clients with STDs (or parents in cases of neonatal eye discharge) were also included. It was assumed that $100 \%$ of such partners are given treatment. 
The following line items were costed:

Training of doctors, nurses, and midwives

On-site training (Facilitative supervision)

IEC activities

Clinic equipment

Equipment maintenance

MIS forms

Costs of treatment

Clinic supplies

\section{Results}

For a clinic without a laboratory, cost per client ranges between $\$ 11$ - $\$ 16$. Seventy percent of this total cost is for diagnosis and treatment alone. This can be explained by the fact that RTI drugs are often costly. Since this study assumes that both the client and her partner complete the treatment regimen, treatment cost thus increases.

Variable costs are at least twice as much as the fixed costs. Thus, what largely determines the overall cost of RTI services in Model 1 is the number of clients served, rather than initial investments in training and equipment.

Using the minimum costs from Table 1, various annual estimates of RTI clients were made. The actual number of clients (226) was increased by $50 \%$ and $100 \%$. The results show that while fixed costs remained the same and variable costs increased, cost per client decreased by $\$ 1$ with a $50 \%$ increase, and by $\$ 2$ when the number of clients was increased another $50 \%$. Thus, in Model 1, cost per client decreases somewhat as more clients are served.

Note, however, that these decreases are actually rather minimal. The reason for this lies in the relatively minor role played by fixed costs in this study. As such, we can say that start-up costs for an RTI management program under this model are fairly small. Conversely, though, we cannot claim that the growth in caseloads over time will result in significant gains in cost per client. 
The following tables summarize the results of the cost analysis.

Table 3

Annual Cost of RTI Case Management at a Clinic Without Laboratory (MODEL 1)

(In Pesos)

\begin{tabular}{|l|c|c|}
\hline \multicolumn{1}{|c|}{ ITEM } & MINIMUM & MAXIMUM \\
\hline Fixed Costs & $\mathbf{2 2 , 5 0 3}$ & $\mathbf{3 0 , 5 6 0}$ \\
Training & 11,220 & 16,700 \\
Supportive Supervision & 2,000 & 3,125 \\
IEC Activities & 1,605 & 1,605 \\
Clinic Equipment & 6,980 & 8,300 \\
Equipment Maintenance & 698 & 830 \\
& & \\
Variable Costs & $\mathbf{7 2 , 5 3 5}$ & $\mathbf{1 1 3 , 0 0 4}$ \\
MIS Forms & 1,308 & 1,588 \\
Treatment cost & 65,567 & 104,266 \\
Clinic Supplies & 5,660 & 7,150 \\
Total Cost & & $\mathbf{1 4 3 , 5 6 4}$ \\
Cost per client & $\mathbf{9 5 , 0 3 8}$ & $\mathbf{6 3 5}$ \\
& $\mathbf{4 2 1}$ & $\mathbf{\$ 1 6} *$ \\
\hline
\end{tabular}

N=226 clients (Partners included for clients with STDs)

$* \$ 1=P 40$

The number of clients (226) is the number of actual RTI cases found in four clinics using syndromic management in Quezon City and Cagayan de Oro City within the six-month observation period.

Table 3.1 Cost for Various Annual Estimates of Patients in Clinics Without Laboratory (MODEL 1) 


\begin{tabular}{|l|l|c|c|c|}
\hline \multirow{4}{*}{ MODEL 1 } & \multicolumn{1}{|c|}{ ITEM } & $\begin{array}{c}\text { ACTUAL } \\
\text { N=226 }\end{array}$ & $\begin{array}{c}\mathbf{5 0 \%} \text { INCREASE } \\
\mathbf{N}=\mathbf{3 3 9}\end{array}$ & $\begin{array}{c}100 \% \\
\text { INCREASE } \\
\text { N=452 }\end{array}$ \\
\cline { 2 - 5 } & Fixed Costs & $\mathbf{2 2 , 5 0 3}$ & $\mathbf{2 2 , 5 0 3}$ & $\mathbf{2 2 , 5 0 3}$ \\
\cline { 2 - 5 } & Variable Costs & $\mathbf{7 2 , 5 3 5}$ & $\mathbf{1 0 8 , 8 0 3}$ & $\mathbf{1 4 5 , 0 7 0}$ \\
\cline { 2 - 5 } & Total & $\mathbf{9 5 , 0 3 8}$ & $\mathbf{1 3 1 , 3 0 6}$ & $\mathbf{1 6 7 , 5 7 3}$ \\
\cline { 2 - 5 } & Cost per Client & 421 & $\mathbf{3 8 7}$ & $\mathbf{3 7 1}$ \\
\cline { 2 - 6 } & & $\$ 11$ & $\$ 10$ & $\$ 9$ \\
\hline
\end{tabular}

$\$ 1=$ P40

Note: Using minimum estimates from Table 1

\section{(2) MODEL 2: CLINIC WITH LABORATORY}

Model 2 describes a main health center that has laboratory capacity. Data and assumptions for Model 2 were generally similar to those for Model 1, except for a few additional items.

In Model 2, the medical technologist assigned in the health center is trained in basic laboratory methods such as gram staining (for gonorrhea, chlamydia, bacterial vaginosis), saline wet mount (trichomoniasis), KOH wet mount (candidiasis) and the VDRL Cards Test (syphilis). A three-day training session held in the laboratory of the nearest tertiary government hospital is provided for. Two important assumptions were made: that a working microscope was already available in the clinic and that a salaried medical technologist had been permanently assigned.

The following items were costed:

- Training of medical technologists

Laboratory equipment

Equipment maintenance

- Costs of treatment

Laboratory diagnosis 
The choice of drugs for the management of RTIs in a clinic with a laboratory is essentially the same as in syndromic management (Model 1). However, since etiologic diagnosis tends to be more specific, multiple drug therapy is often avoided; thus, the treatment costs in this model refer to the specific condition.

The cost of laboratory testing varies according to the infection and the laboratory technique used. Some of the pathogens can be identified by simple microscopic examination of wet mounts while others may require gram stain or some other procedure. Some clients also undergo more than one type of test. As such, costs may vary.

\section{Results}

In a clinic with a laboratory, the total cost is divided almost equally between fixed costs and variable costs. Treatment costs are only $28 \%$ of the total cost, while lab tests account for $17 \%$. The lower treatment cost is due to the specificity of treatment in the etiologic management of RTIs, compared to multiple drug treatments (the so-called "shotgun approach") in syndromic management.

The cost per client for a clinic with a laboratory is between $\$ 17$ - $\$ 25$. The bulk of this total amount is spent on training (29\%) and diagnosis and treatment costs (44\%). Various annual client estimates, at $50 \%$ increase and $100 \%$ increase, were also made for Model 2.

As in Model 1, fixed costs remain the same while variable costs increase with a larger number of clients. As expected, cost per client in Model 2 decreases as well, but the decline is larger than in Model 1. When clients were increased 50\%, cost per client decreased by $\$ 3$ and when clients were increased another $50 \%$, cost decreased by an additional $\$ 1$.

Finally, the study made a comparison of costs between Model 1 and Model 2. To make a valid comparison, the number of clients for both models was kept at 100 . The "standardized" models show the following results in Table 3.

Thus, the cost per client turns out to be $\$ 13.7$ for Model 1 and $\$ 15.3$ for Model 2 . There is only a $\$ 1.6$ difference in the cost per client in a clinic without laboratory and a clinic with laboratory, assuming that each serves 100 clients. This $\$ 1.6$ difference further decreases 
with additional clients. For instance, when the client load is increased to 200, the difference in the cost per client between Model 1 and Model 2 narrows down to \$1.1.

Table 4: Cost of RTI Case Management at a Clinic with Laboratory (MODEL 2)

\begin{tabular}{|c|c|c|}
\hline \multicolumn{3}{|c|}{ (In Pesos) } \\
\hline ITEM & MINIMUM & MAXIMUM \\
\hline Fixed Costs & 26,658 & 35,930 \\
\hline \multicolumn{3}{|l|}{ Training } \\
\hline > Doctors, Nurses, Midwives & 11,220 & 16,700 \\
\hline > Medical Technologists & 4,100 & 5,315 \\
\hline Supportive Supervision & 2,000 & 3,125 \\
\hline IEC Activities & 1,605 & 1,605 \\
\hline Clinic Equipment & 6,980 & 8,300 \\
\hline Laboratory Equipment & 50 & 50 \\
\hline Equipment Maintenance & 703 & 835 \\
\hline Variable Costs & 26,598 & 40,323 \\
\hline MIS Forms & 848 & 1,108 \\
\hline Laboratory diagnosis & 8,788 & 8,788 \\
\hline Treatment cost & 14,902 & 27,459 \\
\hline Clinic Supplies & 2,060 & 2,968 \\
\hline Total Costs & 53,256 & 76,253 \\
\hline \multirow[t]{2}{*}{ Cost per client } & 692 & 990 \\
\hline & $\$ 17 *$ & $\$ 25 *$ \\
\hline
\end{tabular}

$\mathrm{N}=77$ clients (Partners included for clients with $\mathrm{STDs}) * \$ 1=\mathbf{P 4 0}$

Table 4.1 Cost for Various Annual Estimates of Patients in Clinics With Laboratory (MODEL 2)

\begin{tabular}{|l|l|l|l|l|}
\hline & ITEM & ACTUAL & $50 \%$ INCREASE & $100 \%$ \\
\hline
\end{tabular}




\begin{tabular}{|l|l|c|c|c|}
\hline MODEL 2 & & $\mathrm{N}=77$ & $\mathrm{~N}=116$ & $\begin{array}{c}\text { INCREASE } \\
\mathrm{N}=154\end{array}$ \\
\cline { 2 - 5 } & Fixed Costs & 26,658 & 26,658 & 26,658 \\
\cline { 2 - 5 } & Variable Costs & 26,598 & 39,897 & 53,196 \\
\cline { 2 - 5 } & Total & 53,256 & 66,555 & 79,854 \\
\cline { 2 - 5 } & Cost per Client & 692 & 574 & 519 \\
\cline { 2 - 5 } & & $\$ 17$ & $\$ 14$ & $\$ 13$ \\
\hline
\end{tabular}

$\$ 1=\mathrm{P} 40$

Note: Using minimum estimates from Table 2.

Table 5

Comparison of Costs Between Model 1 \&Model 2 for 100 Patients

\begin{tabular}{|l|c|c|}
\hline \multicolumn{1}{|c|}{ ITEM } & $\begin{array}{c}\text { MODEL 1 } \\
\text { (Clinic Without Laboratory) }\end{array}$ & $\begin{array}{c}\text { MODEL 2 } \\
\text { (Clinic With Laboratory) }\end{array}$ \\
\hline Fixed Cost & 22,503 & 26,658 \\
\hline Variable Cost & 32,095 & 34,543 \\
\hline Total & 54,598 & 61,201 \\
\hline Cost per Client & 546 & 612 \\
\hline & $\$ 13.7$ & $\$ 15.3$ \\
\hline \multicolumn{2}{|r|}{ [for 200 clients: } & $\$ 10.8$ \\
\hline
\end{tabular}

$\$ 1=\mathrm{P} 40$

At 100 clients, Model 2 (clinic with laboratory) is higher in fixed costs, mainly on training, and in variable costs, mainly on reagents and lab supplies. As pointed out, the difference in cost per client decreases as more clients are served. 
In summary, what makes syndromic management expensive is the cost of treatment, which expectedly increases with the number of clients. Etiologic management spends less on drugs (since treatment is given specifically for the identified pathogen) and more on training and equipment in the first year of integration. As the number of clients increases, however, the initial investment in fixed costs is offset, at least on a per client basis.

Etiologic management as discussed here, however, consists only of simple microscopy. Laboratory tests have been resorted to by the doctors in this study to confirm initial diagnosis. These tests are often rudimentary. Doctors recognize, for instance, that using gram stain to detect gonorrhea does not give conclusive results. What was usually done was to maximize the laboratory set-up for clinics with laboratory capacity and to use the tests to refine the diagnosis. The use of these tests gave the providers added confidence in managing RTI clients. Nevertheless, further studies need to be made to improve the effectiveness of laboratory tests in identifying the particular RTI and to determine the real value added in using laboratory tests.

Finally, in comparing the two models, it must remembered that marginal costs are computed. Existing resources in both models are therefore not included in the costing. For instance, existing laboratory facilities and equipment as well as the salary of the medical technologist in Model 2 are not costed. The \$1.6 difference between Model 2 and Model 1 assumes therefore two different clinic set-ups. This cost analysis estimates how much it would take to add RTI services in two existing clinic set-ups, one with a laboratory and one without.

To make the integration of RTI services within the local health clinics more sustainable, government officials would do well to maximize the clinic set-up and resources at hand, since, first, there is only a minimal increase in costs for adding RTI services under the laboratory set-up, and, second, RTI diagnosis under this model is likely to minimize the problem of overtreatment. 


\section{CONCLUSIONS AND LESSONS LEARNED}

This study has shown that it is indeed feasible to integrate RTI prevention and management into the FP/MCH program. The intervention utilized to achieve this integration produced an improvement in RTI diagnostic knowledge and skills and the provision of information on RTIs. Client satisfaction with RTI services were substantial and the case management of RTIs was found to be effective. However, the apparent success of the intervention of integrating RTI services within the LGU health center presents numerous challenges to policymakers and program managers.

First of all, partner management remains a difficulty as service providers are not always successful in convincing the client's regular sex partner to come to the clinic for a check-up. The reasons for this can be summarized in four categories: the spouse refuses to have himself examined since he doesn't feel any symptoms; the spouse does not realize he also needs to be treated for the wife's condition to improve; the couple hasn't had sex for a long time, and the spouse thinks treatment is unnecessary; it is embarrassing for male RTI clients to admit they acquired the STD from someone other than their wife or from a commercial sex worker.

Second, sustained RTI drug provision is still a great challenge, especially in a devolved set-up where the local government is responsible for the purchase of drugs. Experience in the study has shown that drug purchase by local governments is often a slow and complicated process. Moreover, some governments simply don't have adequate funding for providing RTI drugs on a regular basis. Hence, further studies are necessary to determine other modes of cost recovery schemes such as charging for drugs through social marketing. The availability of drugs is a major factor in sustaining the integration of RTI services in the health center.

Third, earning the client's trust is important. Good counseling and rapport with clients will help to bring this about. Providers need to be trained in dealing with the sensitive issues posed by RTI management, particularly during risk assessment, giving the key messages; dealing with sensitive information and helping the client to communicate with their partner; and promoting effective partner management. A skilled counselor can get across key messages and information to the client even in a short period of time, which is often key in a public health 
clinic situation.

Corollary to this is the need for ensuring visual and auditory privacy in the clinic and maintaining confidentiality of client information. The situation analyses showed that there were some lapses in maintaining a private area for the PE and counseling of RTI clients. The volume of FP/MCH clients waiting in the health center and the limited time on hand often compromise privacy during consultation. Sometimes the clinic set-up itself is too small or does not lend itself to a private consultation. For instance, in at least one clinic, only a flimsy curtain separated the area for PE and, during one SA observation, other health personnel overheard the conversation between a female RTI client and the doctor. Maintaining visual and auditory privacy in the clinic clearly enhances quality of care.

Fourth, the conditions for referral to a higher center for management of particular RTI cases which cannot be managed at the primary health center need to be defined. Providers need to be guided as to when and where to refer RTI cases, particularly in areas where no doctor is permanently stationed. Referral also pertains to special cases not covered during the training or in the management guidelines.

Moreover, for RTI patients who are currently using a FP method, the syndromic management treatment protocol needs to have special guidelines. Questions such as "when to remove the IUD" and "for what types of RTIs does one remove or not remove the IUD" were often asked by the health workers. In addition, guidelines need to be made on the management of pregnant women with RTI, particularly with regard to choice of drugs, as some might be potentially injurious to the health of the fetus.

One limitation cited about syndromic management that needs to be addressed is its inability to detect RTIs among asymptomatic women. Since, according to literature, half of all women with an RTI fall in this category, these cases will be missed unless a more effective screening procedure is developed. Moreover, since diagnosis can only be made if symptoms fall within a categorical syndrome, other signs and symptoms suggestive of RTIs can also be missed. An example of this symptom is genital itchiness, a condition which can be caused by candidiasis, trichomoniasis, and/or lice infestation. In the present RTI study this was the presenting symptom of 14 percent of all RTI clients. 


\section{Lessons Learned}

The RTI Integration study showed that the majority of the patients do come back for check-up visits. This finding challenges the prevailing presumption that Filipino patients generally do not return after their initial check-up. Within the six months' observation period, seventy-four percent of the clients returned for their follow-up visit with the health workers. The key to convincing RTI clients to return appears to be the consistent message from service providers about the importance of completing treatment and the consequences of noncompliance. The follow-ups made by community health volunteers further bolstered the return rates.

The health providers did not show any indication of resisting RTI integration. The need for post-training facilitative supervision was shown in the study, particularly in areas where client load was high or where the doctor reported only weekly. These supervisory visits can be used by the clinic staff as an occasion to clarify questions about actual RTI case management. The guidance and supervision provided by the physician enable the staff to treat cases more confidently. It was also observed that in the months following the facilitative supervision, RTI caseloads significantly increased in all seven health centers.

Since non-doctors were prohibited from diagnosing and treating RTI clients, a standing order from the medical officer was necessary for nurses and midwives to be able to provide treatment when the doctor was absent. This agreement was reached by the service providers themselves. Most of time, however, it was the nurse, rather than the midwife, who treated RTI clients in the doctor's absence. The doctor then reviewed the case in her/his next visit.

Thus, although syndromic management can be carried out even by non-physicians, many nurses and midwives are not confident in their ability to manage patients because they do not view this as their usual role. This was borne out in the situation analysis findings. As a result, a main lesson learned in this study was the need for supportive supervision to 
overcome this constraint and the need for the physicians to issue a standing order for RTI cases to be managed syndromically by trained staff members in their absence.

In the present study, about a quarter of RTI clients were found to be symptomatic while undergoing the physical examination required of FP clients. The physical exam is a requirement under the $\mathrm{DOH}$ clinical standards for the following cases: new acceptors of any method; within one, three, and six months after insertion of an IUD; and during the annual check-up and Pap smear for current users. Ensuring consistent compliance with this requirement seems to be an efficient and cost-effective strategy for screening RTI asymptomatic women.

The minimal difference in the cost per RTI client in a clinic with laboratory facilities and in a clinic without lab facilities indicates a need to maximize the existing clinic set-up. RTI management with the use of simple lab tests and microscopy appears to be more cost-effective as the number of clients increases. On the other hand, service providers are yet uncertain whether adding simple lab tests and microscopy in the context of the health center improves the effectiveness of RTI case management. Moreover, the need to conduct a validation of syndromic management, which is currently widely used in resource-poor settings, seems to be justified in the Philippine context.

Finally, one way to sustain the supply of drugs in the clinics is to involve LGU officials throughout the process of integration. It was learned in the study that local policymakers were actually concerned about the health and well-being of their constituents. Thus, the provision of RTI drugs has been sustained in areas where the municipal mayor or the city health officer was convinced of the need for integrating RTI services in the primary health center and where they themselves were involved in the sourcing of drugs. 


\section{REFERENCES}

Bang, R.A., et al. 1989. High Prevalence of Gynecological Diseases in Rural Indian Women. The Lancet 1:85-88.

Bhatia, Jagdish C. and John Cleland. 1995. Self-Reported Symptoms of Gynecological Morbidity and Their Treatment in South India. Studies in Family Planning 26:203216.

Cates, Ward. 1996. Recommendations for Updating Selected Practices in Contraceptive Use. Volume 2. 1996.

Department of Health and Acquired Immunodeficiency Syndrome Control and Prevention (AIDSCAP). 1997. STD Case Management Guideline. Manila, Philippines.

Dixon-Mueller, R. and J. Wasserheit. 1991. The Culture of Silence: Reproductive Tract Infections Among Women in the Third World. International Women's Health Coalition, New York.

Iskandar, Meiwita, Subadra Indrawati, Catherine Vickers and Siti Nurul Qomariyah. 1997. Final Report of Activities for "Improved Reproductive Health and STD Services for Women Presenting to Family Planning Services in North Jakarta." Asia and Near East Operations Research and Technical Assistance Project, Population Council, Jakarta.

Janowitz, Barbara and John H. Bratt. 1994. Methods for Costing Family Planning Services. UNFPA and Family Health International.

Khan, M.E., Saumya RamaRao and John W. Townsend. 1996. A Model of Costs for RTI Case Management Services in Uttar Pradesh (Technical Paper). Asia and Near East Operations Research and Technical Assistance Project, Population Council, New 
Delhi.

Kisubi, W., Francesta Farmer and Richard Sturgis. 1997. An African Response to the Challenge of Integrating STD/HIV-AIDS Services Into Family Planning Programs. Pathfinder International, Massachusetts.

Mugrditchian, Doris S. 1996. Update on RTI Diagnostics and Antibiotics Resistance. Report presented at the Regional Meeting on Reproductive Tract Infections. February 22-24, 1996, Bangkok, Thailand.

National Statistics Office (NSO) [Philippines] and Macro International Inc. (MI). 1994a. National Safe Motherhood Survey 1993. Calverton, Maryland: NSO and MI.

------- and ------. 1994b. National Demographic Survey 1993. Calverton, Maryland: NSO and MI.

Pachauri, Saroj. 1995. Defining A Reproductive Health Package for India: A Proposed Framework. Regional Working Paper No. 4, Population Council, New Delhi.

Quintong, Jovencia. 1996. "From Family Planning to Reproductive Health: A Program Manager's Perspective." Paper presented at the Operations Research Training Workshop for Program Managers and Researchers, organized by the Department of Health and Population Council, Manila, January 15-21, 1996, Puerto Galera, Philippines.

Sanchez, Rosena and Maribeth P. Juarez. 1994. Community-Based Research and Advocacy in Reproductive Health Among Urban Poor Women in Davao City. Development of People's Foundation, Davao City, Philippines.

Twahir, Amina, Baker Ndugga Maggwa and Ian Askew. 1996. Integration of STI and HIV/AIDS Services with MCH-FP Services: A Case Study of the Mkomani Clinic Society in Mombasa, Kenya. Operations Research and Technical Assistance Africa Project II, Population Council.

Wasserheit, Judith N., et al. 1989. Reproductive Tract Infections in a Family Planning Population in Rural Bangladesh. Studies in Family Planning 20:69-80.

Wi, Teodora Elvira. 1997. Report on Project Activities on Sexually Transmitted Diseases in the Philippines. AIDSCAP and the University of Washington. Manila, Philippines. 
World Health Organization Regional Office for the Western Pacific Region. 1997. STD Case Management: The Syndromic Approach for Primary Health Care Settings. Manila, Philippines.

Younis, Nabil, et al. 1993. A Community Study of Gynecological and Related Morbidities in Rural Egypt. Studies in Family Planning 24: 175-186. 